\title{
Utility of Global Ensemble Forecast System (GEFS) Reforecast for Medium-Range Drought Prediction in India $\mathscr{O}$
}

\author{
REEPAL D. SHAH AND VIMAL Mishra \\ Civil Engineering, and Information Technology Research Academy Water Project: Measurement \\ to Management (M2M), Indian Institute of Technology Gandhinagar, Gujarat, India
}

(Manuscript received 7 April 2015, in final form 29 March 2016)

\begin{abstract}
Medium-range ( $\sim 7$ days) forecasts of agricultural and hydrologic droughts can help in decision-making in agriculture and water resources management. India has witnessed severe losses due to extreme weather events during recent years and medium-range forecasts of precipitation, air temperatures (maximum and minimum), and hydrologic variables (root-zone soil moisture and runoff) can be valuable. Here, the skill of the Global Ensemble Forecast System (GEFS) reforecast of precipitation and air temperatures is evaluated using retrospective data for the period of 1985-2010. It is found that the GEFS forecast shows better skill in the nonmonsoon season than in the monsoon season in India. Moreover, skill in temperature forecast is higher than that of precipitation in both the monsoon and nonmonsoon seasons. The lower skill in forecasting precipitation during the monsoon season can be attributed to representation of intraseasonal variability in precipitation from the GEFS. Among the selected regions, the northern, northeastern, and core monsoon region showed relatively lower skill in the GEFS forecast. Temperature and precipitation forecasts were corrected from the GEFS using quantile-quantile (Q-Q) mapping and linear scaling, respectively. Biascorrected forecasts for precipitation and air temperatures were improved over the raw forecasts. The influence of corrected and raw forcings on medium-range soil moisture, drought, and runoff forecasts was evaluated. The results showed that because of high persistence, medium-range soil moisture forecasts are largely determined by the initial hydrologic conditions. Bias correction of precipitation and temperature forecasts does not lead to significant improvement in the medium-range hydrologic forecasting of soil moisture and drought. However, bias correcting raw GEFS forecasts can provide better predictions of the forecasts of precipitation and temperature anomalies over India.
\end{abstract}

\section{Introduction}

Agriculture is a lifeline for the Indian population and accounts for $17 \%$ of the nation's gross domestic product (GDP). Approximately $51 \%$ of the workforce in India is associated with agriculture, which is often impacted by short-term abnormal hydroclimatic conditions. For instance, severe drought in 2002 resulted in substantial economic losses, and unexpected precipitation events in March 2015 caused enormous damage to crops that were about to be harvested in various parts of India. A

Supplemental information related to this paper is available at the Journals Online website: http://dx.doi.org/10.1175/JHM-D-15-0050.s1.

Corresponding author address: Vimal Mishra, Civil Engineering, Indian Institute of Technology Gandhinagar, VGEC Campus, Chandkheda, Ahmedabad, Gujarat 382424, India.

E-mail: vmishra@iitgn.ac.in hailstorm in Maharashtra in 2014 damaged crops in 0.67 million ha and had severe repercussions for the socioeconomic conditions of farmers in the region. Climate and weather anomalies often lead to economic losses in the farming community in India.

There have been several efforts to provide weekly monitoring of the impacts of weather and climate anomalies on agriculture and water resources in India. For instance, the National Centre for Medium Range Weather Forecasting (NCMRWF) was established in India with the aim to reduce agro-economic losses due to the short-term variability of weather. The NCMRWF aims to assist farmers in decision-making during farm operation and at various crop-growth stages (Kumar et al. 2000; Saseendran et al. 2002; Rathore and Maini 2008; Sikka 2009). For instance, farmers of the state of Tamil Nadu were informed for early sowing in September 1995 based on rainfall forecast from the NCMRWF. Timely rainfall forecast 
at key crop-growth stages helped farmers to save irrigation water and increase profit in Raipur, Chhattisgarh (Rathore and Maini 2008). Since the intraseasonal variability (ISV) in rainfall is high, weekly forecasts of precipitation and other hydrologic variables can be valuable for decision-making in agriculture. There have been several efforts for the weekly monitoring of soil moisture and droughts in many regions. For instance, Palmer (1968) developed a crop moisture index for weekly monitoring of the impact of drought on crop yields in the United States. He showed that variability in crop yields could be described by a weekly crop moisture index, which is based on weekly conditions of vegetation and meteorological variables. Moreover, Narsimhan and Srinivasan (2005) demonstrated that a weekly drought index based on soil moisture can provide valuable information on crop yields at various crop-growth stages. Shah and Mishra (2015) developed an experimental drought monitoring system that updates daily and provides real-time information on hydrologic variables at $0.25^{\circ}$ resolution.

Medium-range forecasts may tend to have noise and bias that may affect hydrologic forecasting. Statistical postprocessing is helpful in improving the forecast of meteorological variables and in enhancing skill of hydrologic prediction (Hamill et al. 2013). The World Meteorological Organization (2012) documented many techniques to postprocess forecasts for improving prediction skill. Fan and Dool (2011) corrected weekly predictions from the Global Ensemble Forecast System (GEFS) using bias during the 7-30 days leading up to the date of the forecast with an aim to improve simulations of soil moisture in the United States. However, they found that the improvement after the bias correction was modest. Gutiérrez et al. (2004) demonstrated a clustering algorithm for statistical downscaling ensemble members of the short-range forecast from the European Centre for MediumRange Weather Forecasts (ECMWF). Other studies have used dynamical downscaling with some success to postprocess meteorological model forecasts; for example, Misra et al. (2003) improved seasonal forecast from global climate models (GCMs). The quantilequantile $(\mathrm{Q}-\mathrm{Q})$ mapping approach can improve the skill in the variables that show strong correlations between observations and predictions (Abraham et al. 2013); however, if a variable (e.g., precipitation) has a poor correlation, the $\mathrm{Q}-\mathrm{Q}$ mapping may lead to errors (Madadgar et al. 2014).

Postprocessed medium-range ( $\sim 7$ days) forecasts of precipitation and temperature forced with a wellparameterized hydrologic model may provide estimates of root-zone $(\sim 60 \mathrm{~cm})$ soil moisture and runoff. Skillful medium-range weather forecasts can be used to improve the skill of seasonal predictions of soil moisture and runoff (Shukla et al. 2012). Most of the skill for a medium-range forecast of simulated soil moisture may come from initial hydrologic conditions (IHCs; i.e., the initial state of soil moisture; Shukla and Lettenmaier 2011), which can be attributed to soil moisture persistence. After IHCs, a major contribution to seasonal soil moisture forecast skill beyond 1-month lead comes from atmospheric forcing (precipitation and air temperature). Shukla and Lettenmaier (2011) reported that over the equatorial humid regions and the monsoonal regions, the contribution from atmospheric forcing is higher than that of IHCs throughout the year (Shukla et al. 2013). Precipitation forecasts may provide higher skill for agricultural drought prediction during periods when the persistence in soil moisture is low and variability in precipitation is high. As mentioned above, timely precipitation and hydrologic forecast can be valuable for water management and reduction of economic losses.

A medium-range ( $\sim 7$ days) forecast is needed for better decision-making in the agriculture and water resources sector in India. The overarching science question we aim to address is if weather reforecasts can provide skill to hydrologic forecasts for weekly drought predictions. The outcome of this paper is to develop a medium-range drought prediction system using observations from the India Meteorological Department (IMD), forecasts from the GEFS reforecast (Hamill et al. 2013), and the Variable Infiltration Capacity model (VIC; Liang 1994) for India. To do so, we used ensemble mean forecasts of precipitation and maximum and minimum temperatures at a resolution of T382L64 (horizontal resolution $\sim 35 \mathrm{~km}$ and 64 sigma hybrid pressure levels) to force the hydrologic model (at daily temporal and $0.25^{\circ}$ spatial resolution) and derive information on medium-range predictions (1-7-day leads) of soil moisture and runoff for the period of 1985-2010. Ashrit et al. (2013) evaluated the performance of the GEFS at T190L28 resolution for the monsoon season in the year 2012 over India. They found an improvement in T190L28 GEFS when compared to operational deterministic fineresolution T574 forecasts for 10 days lead time. In this paper, we evaluate the retrospective reforecasts from the GEFS against the observed precipitation and temperature data from the IMD to understand the skill of the medium-range forecast. The mediumrange drought prediction system aims to provide the predictions of root-zone soil moisture, total runoff, precipitation, and air temperature for the entire domain of India at $0.25^{\circ}$ spatial resolution on a daily basis. 


\section{Data and methods}

\section{a. GEFS}

We used precipitation and temperature forecasts from the GEFS reforecast (Hamill et al. 2013) for mediumrange drought predictions in India. Initial conditions for the forecast in the Global Forecast System (GFS; the numerical weather prediction model in GEFS) model are derived from the Climate Forecast System Reanalysis (CFSR; Saha et al. 2010), up to 20 February 2011. Shah and Mishra (2014) evaluated precipitation and air temperature from the CFSR for the monsoon season droughts in India and found that the CFSR showed a better performance in capturing droughts (based on precipitation and evapotranspiration deficits) than the other modern-era reanalysis products (MERRA and ERA-Interim). In the GEFS reforecasts, perturbations are made in initial atmospheric conditions to generate the ensemble members of the forecast. The perturbed initial conditions are estimated using an ensemble transform technique with rescaling (ETR; Wei et al. 2008). For the operational real-time forecast, 80 members are used for generating initial conditions, out of which 20 leading conditions are used to initialize the operational forecast up to 16 days lead. The operational forecasts are generated at every $6 \mathrm{~h}$ from $0000,0600,1200$, and 1800 UTC initial conditions. The reforecasts are generated daily at 0000 UTC using only 10 perturbations and a single control initial condition. For the first eight forecast days, forecasts are run at T254L42 resolution, which is equivalent to $40 \mathrm{~km}$ at $40^{\circ}$ latitude and 42 vertical levels (Hamill et al. 2013). At lead day +7.5 , forecasts are integrated at T190L42, which is approximately $54 \mathrm{~km}$ at $40^{\circ}$ latitude, and forecast products are saved at this resolution from +8 to +16 lead days. Since we were interested in the skill of the higher-resolution forecast for hydrologic applications and the skill will also drop with increase in lead time, we limited our analysis to 1-7-day lead times.

The GEFS reforecast is available at $1^{\circ}$ resolution at every 3 -h interval for $0-72 \mathrm{~h}$ and every 6 - $\mathrm{h}$ interval for $72-384 \mathrm{~h}$. We aggregated $1^{\circ}$ daily precipitation and maximum (Tmax) and minimum (Tmin) temperatures from 3-hourly forecasts for the period 1985-2010.

\section{b. Observed precipitation and air temperatures}

Daily precipitation and maximum and minimum temperature data from the IMD were used to evaluate the skill of the GEFS forecasts at $0.25^{\circ}$ spatial resolution and to generate IHCs (as direct measurements of IHCs are limited) using the hydrologic model. The IMD precipitation product was developed for the period of 19012010 using observations from 6995 meteorological stations across India through the inverse distance weighted scheme (Pai et al. 2014). The new IMD precipitation dataset is quality controlled and has been used in previous studies (Mishra et al. 2014; Shah and Mishra 2015). Mishra et al. (2014) used the gridded IMD precipitation data to evaluate hydrologic changes under the retrospective (1950-2008) climate in India.

We obtained gridded (at $1^{\circ}$ ) daily maximum and minimum temperature data from the IMD for the period of 1951-2010 (Srivastava et al. 2009). The gridded temperature data were based on observations from 395 stations across India. The density of temperature measurement stations is lower in the northern regions in India, which may cause bias and uncertainty in the gridded product, as mentioned in Shah and Mishra (2015) and Mishra (2015). To make the coarseresolution data consistent with the spatial resolution of the experimental drought monitor (Shah and Mishra 2015), daily maximum and minimum temperature data were interpolated to $0.25^{\circ}$ spatial resolution using the synergraphic mapping system (SYMAP) described in Maurer et al. (2002). Observed (OBS) precipitation and temperature data from the IMD were used to develop the experimental drought monitor for India as described in Shah and Mishra (2015). Since the GEFS reforecast is available from 1985 onward, we limited our analysis to the period of 1985-2010 (26 years), which is common in both observations (IMD) and the GEFS forecasts. To evaluate the GEFS forecasts, we obtained the ensemble mean forecast for the 1-7-day lead time for precipitation and minimum and maximum temperatures. Then, the 1-7-day-lead-time forecast was aggregated to a weekly time scale for both precipitation and air temperatures. Since our aim was to provide the forecast for 7 days lead time, we evaluated the GEFS reforecast for accumulated precipitation and average temperatures.

\section{c. Postprocessing of the GEFS forecast}

We used the Q-Q mapping [also referred to as cumulative distribution function (CDF) matching; Wood et al. 2002] approach to improve the Tmax and Tmin forecasts from the GEFS. We developed cumulative distribution functions for weekly averaged air temperatures from the GEFS reforecast and OBS for each day of the forecast (considering Julian days). We developed CDFs using the empirical Weibull plotting position where percentiles fall between $1 /(N+1)$ and $N /(N+1)$, where $N$ is number of climatological years used to derive percentile. For values beyond the given range, a normal distribution was adopted as suggested in Wood et al. (2002). We used multifold cross validation by randomly selecting 25 years for training and leaving 1 year for testing, which resulted in 26 combinations of training and testing periods. One of the assumptions associated 
with the Q-Q mapping is that the distributions involved in the mapping are representative throughout the period of the analysis.

We evaluated linear scaling to correct bias in accumulated precipitation from the GEFS reforecast for 7-day lead times. To apply the linear scaling method for each grid cell, we initially corrected extreme events (above the 90th percentile) of the distribution at a given day of year during a training period ( 25 years). To do so, first we estimated all the forecast years that have weekly accumulated precipitation values above the 90th percentile on the considered day of forecast. Total weekly precipitation from these extreme events was compared with the total precipitation from OBS for corresponding years, and scaling factors (based on ratios of precipitation from OBS and the GEFS) were obtained. Based on scaling factors, bias in extreme precipitation was corrected (by multiplying with the scale factors). After this, we estimated the scaling factor for total weekly precipitation from the GEFS and OBS, which was used to correct the precipitation data from the GEFS. This technique is similar to that used in Shah and Mishra (2015) to correct observed precipitation from the Tropical Rainfall Measuring Mission (TRMM) satellite. Then scaling factors were evaluated for the testing period (1 year). Similar to temperatures, multifold cross validation was performed for precipitation using randomly selected 25 years for training and 1 year for testing.

\section{d. The VIC}

The VIC (Liang 1994; Liang et al. 1996; Cherkauer et al. 2003) was used to simulate soil moisture and total runoff for the period of 1985-2010. The VIC is a semidistributed macroscale hydrologic model. Subgrid variability and spatial heterogeneity in vegetation, soil, and topography can be well represented in the VIC. Evapotranspiration in the VIC is estimated using the Penman-Monteith equation (Shuttleworth 1999). The VIC has been applied for hydroclimatic studies at regional and national scales at various spatial resolutions ranging from more than 1 to $100 \mathrm{~km}$ (Maurer et al. 2002; Nijssen et al. 2001). The VIC was successfully implemented in the Indian region for soil moisture drought assessment (Mishra et al. 2014) and for the development of the real-time drought monitoring system (Shah and Mishra 2015). More information on the VIC can be obtained from Gao et al. (2009).

We applied the VIC (version 4.1.2) in water balance mode to run daily simulations for the period of 19852010. We considered three soil layers: the top layer was fixed to $10 \mathrm{~cm}$ and the depth of the second and third layers were obtained through calibration as explained in
TABLE 1. Experiments to understand the role of bias-corrected forcings on soil moisture and total runoff forecast.

\begin{tabular}{cll}
\hline \hline No. & \multicolumn{1}{c}{ IHCs } & \multicolumn{1}{c}{ Forcing (1-7 day lead) } \\
\hline 1 & Retro simulated (SIM) & Observed (OBS) \\
2 & SIM & Raw GEFS (RAW) \\
3 & SIM & Corrected GEFS (Co_GEFS) \\
\hline
\end{tabular}

Mishra et al. (2014) and Shah and Mishra (2015). The vegetation parameters were developed using 1-km Advanced Very High Resolution Radiometer (AVHRR) global land-cover information (Hansen et al. 2000). Soil parameters were developed using the Harmonized World Soil Database (HWSD) project using the method described on the Land Surface Hydrology website at the University of Washington. The soil parameters were obtained from the previous studies for the VIC implementation in India (Mishra et al. 2014; Shah and Mishra 2015). The parameters and the model setup have also been evaluated against the observed streamflow and soil moisture in India (Mishra et al. 2014; Shah and Mishra 2015).

We designed experiments using the VIC to understand the influence of bias correction of the GEFS forecasted forcings on medium-range drought prediction. For each initialization day of the hydrologic forecast, we obtained the IHCs by running the VIC up to the initialization date using the observed IMD forcings. From the initialization date, the VIC was then run using the corrected and raw (interpolated from $1^{\circ}$ to $0.25^{\circ}$ ) GEFS-based forcing to evaluate the effectiveness of bias correction as described in Table 1.

\section{e. Soil moisture, streamflow, and drought observations}

The role of bias correction was evaluated against the soil moisture from satellite and observed streamflow for the Narmada River basin at Garudeshwar (Garud) gauge station $\left(73.65^{\circ} \mathrm{N}, 21.89^{\circ} \mathrm{E}\right)$. Satellite-based soil moisture was obtained from the European Space Agency Climate Change Initiative (ESACCI; Dorigo et al. 2012) and observed streamflow was obtained from the Water Resources Information System of India (WRIS; www.india-wris.nrsc.gov.in). The drought forecast was evaluated using the satellite-driven 8-day composite drought severity index (DSI) as described in Mu et al. (2013), which is based on vegetation health and evapotranspiration (ET) from the Moderate Resolution Imaging Spectroradiometer (MODIS). The DSI combines the normalized difference vegetation index (NDVI) and the ratio of evapotranspiration to potential evapotranspiration (PET) into a single index. Further information on DSI can be obtained in Mu et al. (2013). 

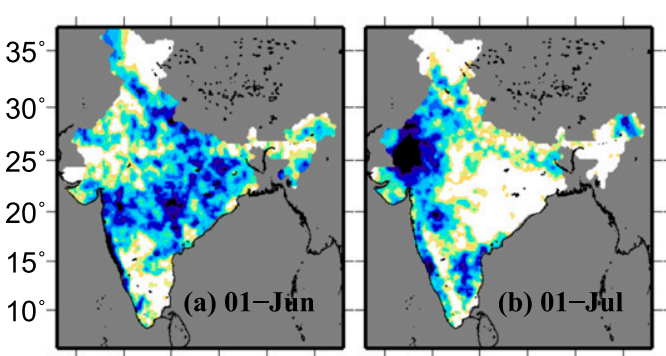

\section{Monsoon}
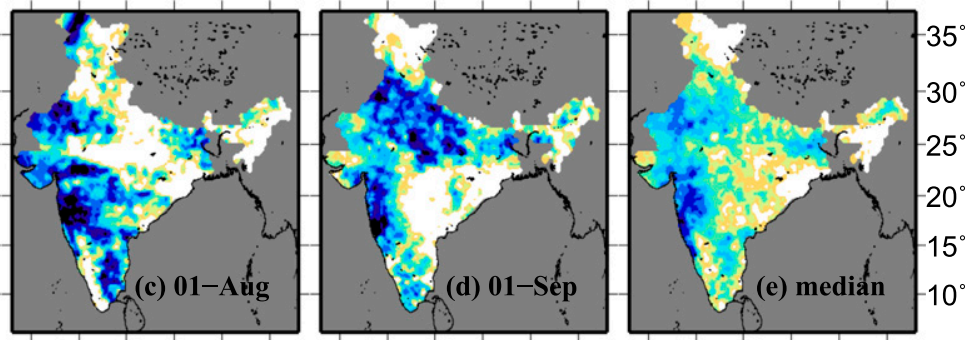

Winter
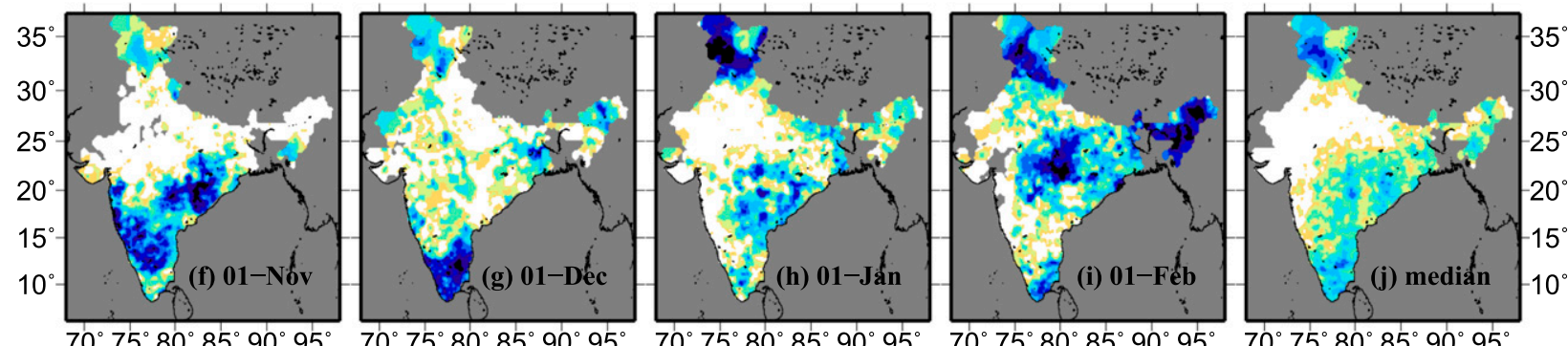

$70^{\circ} 75^{\circ} 80^{\circ} 85^{\circ} 90^{\circ} 95^{\circ}$

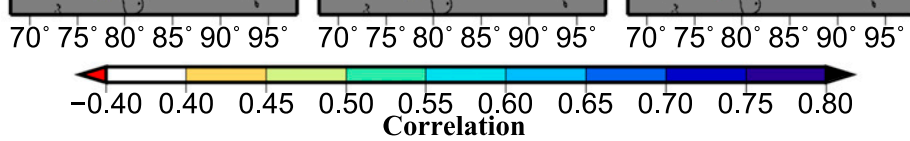

$70^{\circ} 75^{\circ} 80^{\circ} 85^{\circ} 90^{\circ} 95^{\circ}$

Correlation

FIG. 1. Correlation between weekly accumulated precipitation predictions from GEFS and OBS (weekly accumulated for the same dates). Weekly predictions were correlated for first day of each (a)-(d) monsoon and (f)-(i) winter season (i.e., nonmonsoon) month. (e),(j) Median of correlations estimated on the first day of each monsoon and winter month. Correlations were estimated for the period of $1985-2010$.

We also evaluated soil moisture and runoff simulations obtained using the GEFS forecast against those that were simulated using the observed forcing from the IMD. Daily wind speeds were obtained from Sheffield et al. (2006) to run the VIC. Initial hydrologic conditions to force VIC using forecasts were generated for 2 days (14 February and 14 July) each year during the period of 1985-2010 (26 years). We used only these two dates to evaluate the GEFS forecast skill in simulating soil moisture and total runoff in the monsoon and nonmonsoon seasons to make it computationally economical. However, we evaluated the forecast skill of precipitation and daily maximum and minimum temperature for the entire year. For each run, we provided an appropriate (more than 5 years) spinup period to the VIC to avoid any inconsistency in simulations of soil moisture and total runoff.

\section{Results and discussion}

\section{a. Evaluation of precipitation and temperature forecast skill for the retrospective period (1985-2010)}

We evaluated 7-day accumulated anomaly correlations between OBS and the GEFS precipitation for the monsoon and nonmonsoon seasons (Fig. 1). The evaluation was performed for the retrospective period of 1985-2010.
For both monsoon and nonmonsoon seasons, we considered the first day of the month as a day of the forecast. We find that during the monsoon season, anomaly correlation varies spatially and temporally. For instance, for the forecasts initiated on 1 June, strong correlation skill was observed in central India (Fig. 1a). On the other hand, correlations were weaker in the southern peninsula and western India. As the monsoon season progresses, correlations between accumulated precipitation from the OBS and the GEFS diminish in the core monsoon region located in east-central India. This feature is consistently evident in the months of July-September (Figs. 1b-d). Median correlations for the monsoon season also exhibit a weak relationship in the core monsoon region, whereas there is a slightly better skill in semiarid western India (Fig. 1e). Weaker skill of the GEFS forecast in the core monsoon region can be largely attributed to high ISV in the region, resulting from active-break periods ranging normally around 3-7 days (Rajeevan et al. 2010). The ISV in the GEFS forecast skill can also be noticed during the nonmonsoon season. Here, it should be noted that precipitation totals during the nonmonsoon season are far lower than those of the monsoon season. The GEFS forecast showed stronger skill in the southern peninsula for forecasts initiated on 1 November and 1 December and in central India during the month of February (Figs. 1f-j). 

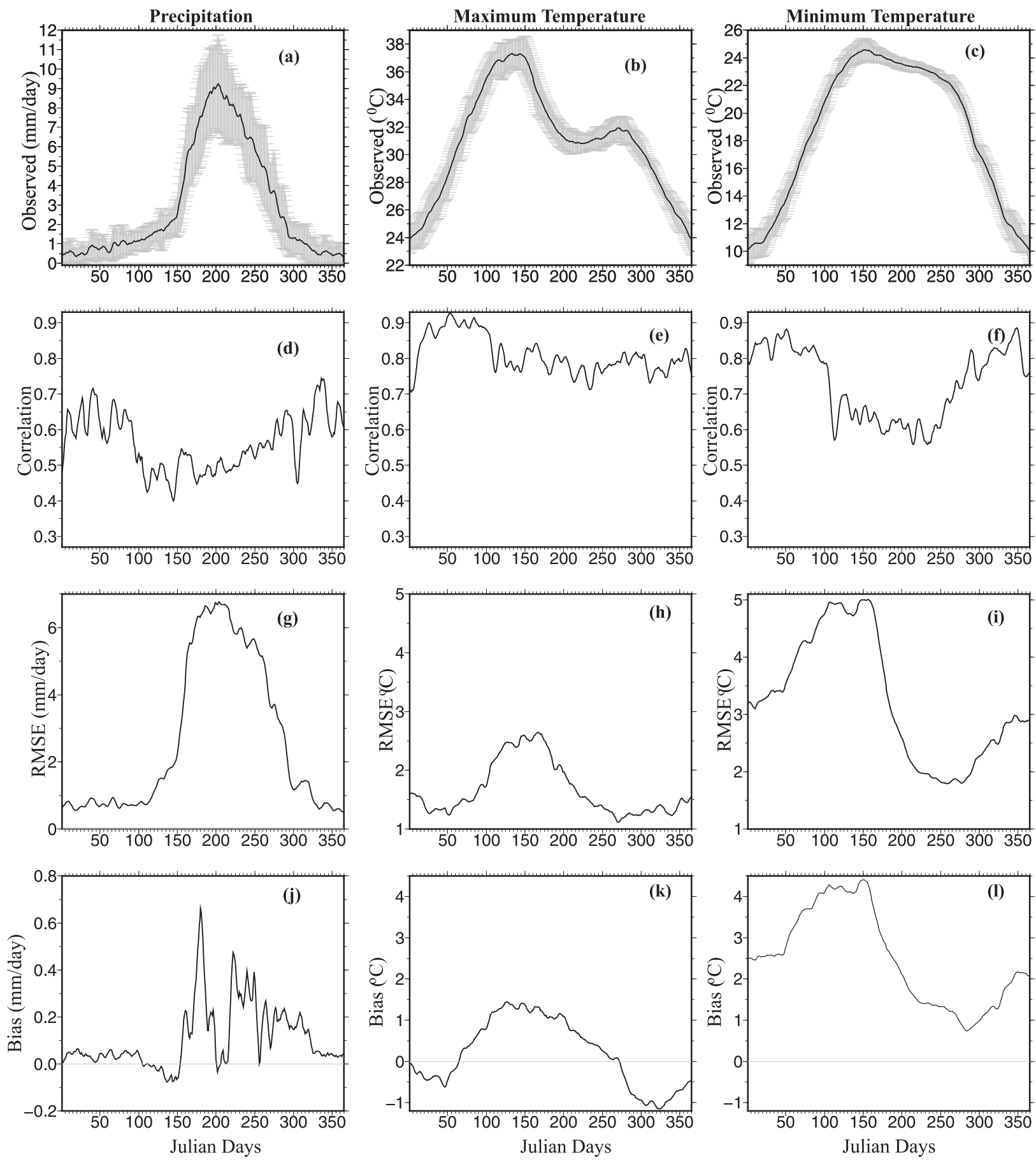

FIG. 2. (a)-(c) Mean area-weighted 7-day-lead accumulated precipitation $\left(\mathrm{mm}\right.$ day $\left.{ }^{-1}\right)$, $\operatorname{Tmax}\left({ }^{\circ} \mathrm{C}\right)$, and Tmin $\left({ }^{\circ} \mathrm{C}\right)$ from $\mathrm{OBS}(\mathrm{dark}$ line) estimated on each Julian day. Shaded area shows standard deviation during the period 1985-2010. (d)-(f) All-India median correlation between weekly accumulated predictions of precipitation, Tmax, and Tmin from the GEFS reforecast, version 2, with corresponding accumulated OBS estimated on each Julian day. (g)-(i) RMSE in precipitation $\left(\mathrm{mm} \mathrm{day}^{-1}\right)$, Tmax $\left({ }^{\circ} \mathrm{C}\right)$, and $\operatorname{Tmin}\left({ }^{\circ} \mathrm{C}\right)$ from the GEFS as compared to OBS. (j)-(l) Bias in precipitation $\left(\mathrm{mm}\right.$ day $\left.{ }^{-1}\right)$, Tmax $\left({ }^{\circ} \mathrm{C}\right)$, and Tmin $\left({ }^{\circ} \mathrm{C}\right)$ from the GEFS as compared to OBS. Statistics on each Julian day are 7-day moving mean.

Figure 2 shows observed climatology and skill of reforecast based on the weekly accumulated precipitation and mean temperatures for the forecast dates. Figures 2a-c show all-India mean (1985-2010) and interannual variability of OBS precipitation and temperatures during the forecast weeks. OBS precipitation showed higher interannual variability during the monsoon months and temperatures during summer months. 
Performance statistics including the Spearman rank correlation, root-mean-square error (RMSE), and bias were estimated against OBS weekly accumulated precipitation and mean maximum and minimum temperatures. Based on all-India median statistics, we find that the GEFS forecasts have higher skill in precipitation during the nonmonsoon season and especially in the winter months (Figs. 2d,g,j). Lower skill during the monsoon season (June-September) can be attributed to the lesser ability of GEFS in capturing intraseasonal and interannual variability in precipitation and to total precipitation (Chaudhari et al. 2013; Durai and Bhowmik 2014). This may be because of the lesser skill of GEFS in capturing active-break phase (Rajeevan et al. 2010) during the monsoon season.

The mean maximum and minimum temperatures from the GEFS forecasts showed a higher correlation with OBS in the premonsoon period (January-May) than that of the monsoon season (Fig. 2e). The difference in skill between maximum temperature in the premonsoon and monsoon seasons can be attributed to the effectiveness of the monsoon season precipitation simulations in GEFS. In general, there is a strong negative relationship between the monsoon season precipitation and air temperature, and bias in precipitation can result in bias in air temperature simulations (Shah and Mishra 2014). We find a negative bias in maximum temperature from GEFS during the winter season and positive bias in the monsoon season (Fig. 2k). Similar to mean maximum temperature, higher skill can be observed in the mean minimum temperature forecast during the nonmonsoon season (Figs. 2f,i,l). However, unlike mean maximum temperature, the bias in mean minimum temperature in the GEFS forecast was predominantly positive (Fig. 21).

Figure 3 shows spatial variability (box plot shows statistics of all the grid cells in each region) of skill (correlation, RMSE, and bias) in the selected regions across India. Correlations between accumulated precipitation from GEFS and OBS were lower for the northern and northeastern regions during the monsoon season (Fig. 3b). Correlations generally improve for all the regions during the nonmonsoon season, but with a large spatial variation in skill within each region, as shown by the whiskers of box plots (Fig. 3c). Skill in general improved in all the regions in the nonmonsoon season, indicating that the GEFS forecast performed better in the nonmonsoon season than that of the monsoon season. The difference in the GEFS performance in the monsoon and nonmonsoon seasons can be explained because of limitations in the ability of GEFS to represent key modes of variability that contribute to ISV (see Figs. 1-3 in the supplemental material). Variability within the monsoon season is remarkably high because of large-scale and local processes during the active and break phases of the monsoon. Moreover, it should be noted that the majority of the regions in India receive more than $80 \%$ of the total annual precipitation during the monsoon season. Intraseasonal variability of precipitation is higher in the northcentral, northeastern, and northern regions (Krishnamurthy and Shukla 2008).

To understand the dominant modes of ISV in precipitation, we performed empirical orthogonal function (EOF) analysis [see Mishra et al. (2012) for more details] using pentads (5-day accumulated) of precipitation from the IMD and GEFS (at lead 1 day) for the period of 1985-2010. We selected the pentad for analysis rather than weekly accumulated precipitation, as pentads are commonly used for the active and break analyses during the monsoon season (Kessler 2001). Since the ISV is high during July and August, we limited our analysis only for these 2 months. Figure 1 in the supplemental material shows the first four modes obtained using the EOF analysis and their corresponding principal components (PCs) for the IMD and GEFS precipitation. The correlation between first four PCs from IMD and GEFS was $0.72,0.68,0.47$, and 0.47 , respectively (Fig. 1 in the supplemental material). Results showed that the GEFS fails to represent the spatial variability of the leading modes in peninsular and central India as well as in the foothills of the Himalayas.

Since the monsoon season wind is one of the major factors leading to active and break cycles (Mishra et al. 2012), which in turn result in ISV, the EOF analysis was performed for the 850-hPa wind fields (horizontal $u$ and vertical $v$ ) obtained from the GEFS and the ERAInterim data (Dee et al. 2011) for July-August for the period of 1985-2010. Similar to the EOF analysis conducted for the precipitation, pentad data of wind fields were used for the EOF analysis. Disparities in the first mode obtained from the EOF analysis are associated with the differences in spatial variability in precipitation in the foothills of the Himalayas (Figs. 2a,c in the supplemental material). Similarly, differences in the third and fourth modes obtained from the EOF analysis of the $850-\mathrm{hPa}$ wind fields are linked with the ability of the GEFS to capture spatial variability of precipitation in the central and southern peninsula (Fig. 3 in the supplemental material). These results highlight that accurate initial conditions in the GEFS may lead to improvements in the medium-range forecast because of better representation of ISV, which is consistent with the findings of Chaudhari et al. (2013).

Regional differences in forecast skill of maximum temperature from GEFS are presented in Fig. 4. In comparison to the monsoon/nonmonsoon season precipitation, GEFS 

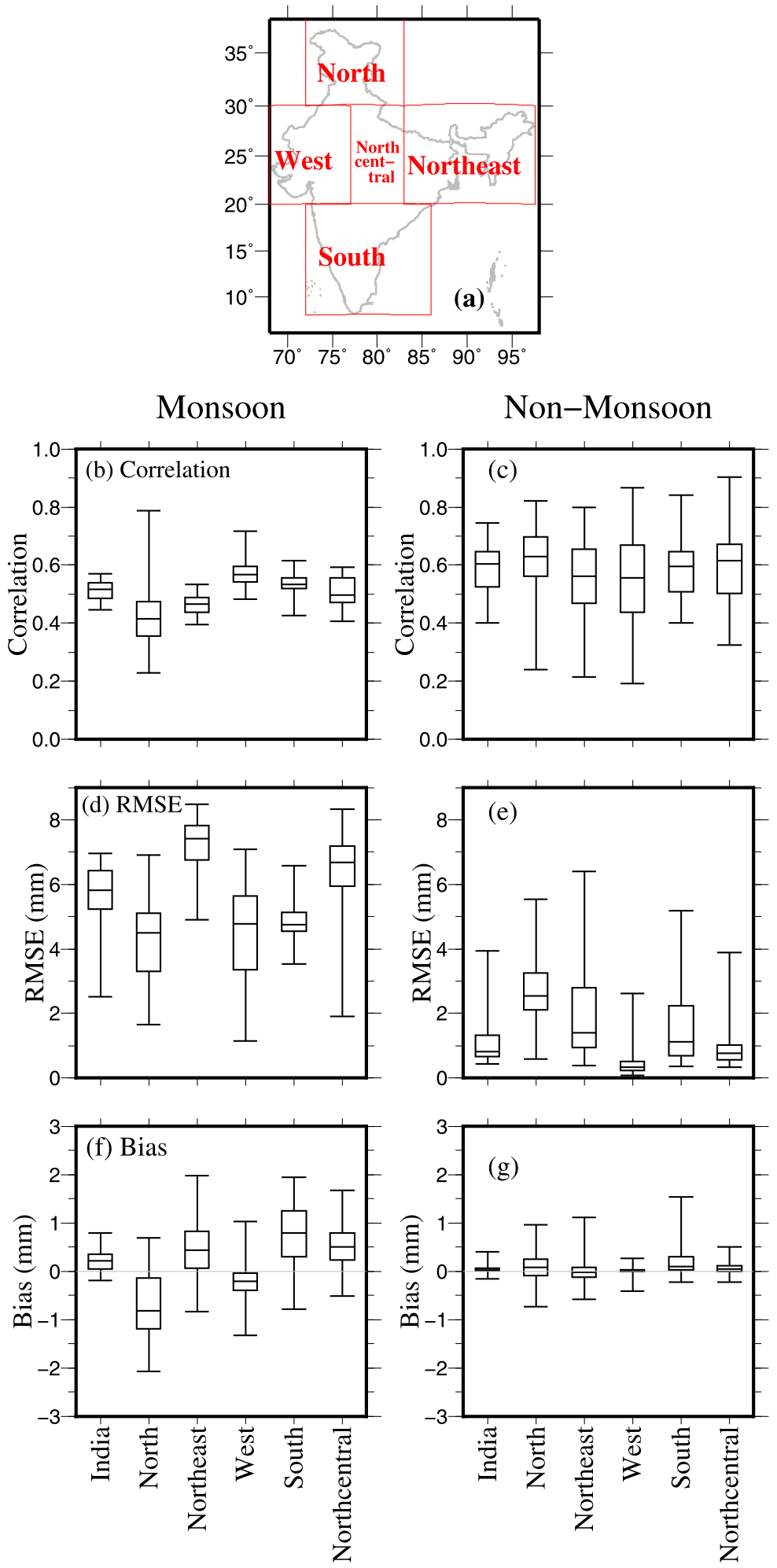

FIG. 3. (a) Geographical areas of the selected regions that were considered for the evaluation of forecast skill and (b),(c) regionwise box plot of correlation between weekly accumulated predictions of precipitation from GEFS and OBS during the monsoon (June-September) and nonmonsoon seasons (October-May). (d),(e) RMSE (mm) and (f),(g) bias (mm) in the GEFS forecast as compared to OBS. Box plots are based on median and 0, 25th, 75th, and 100th percentiles. The 7-day moving mean correlation was used instead of daily correlation. 

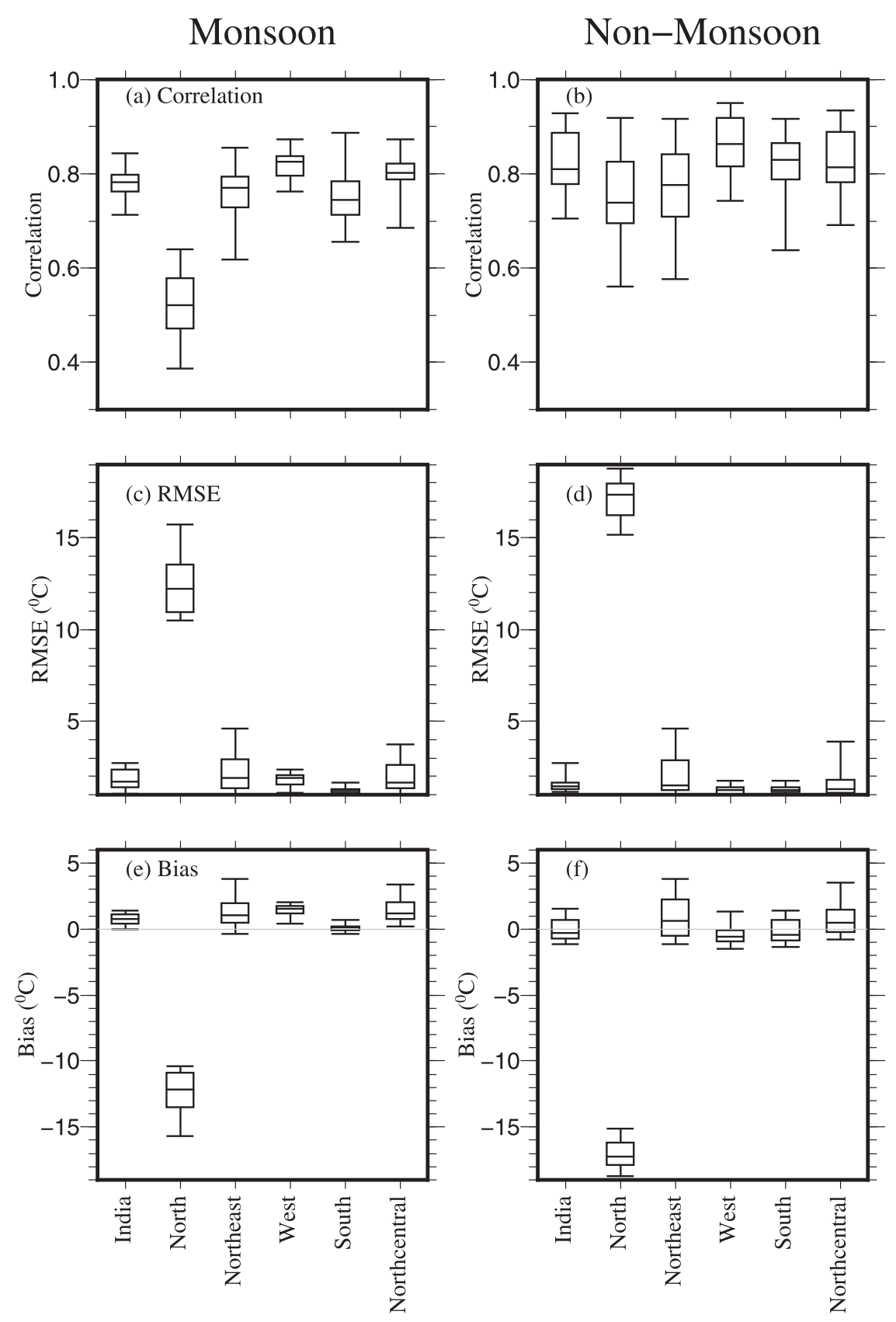

FIG. 4. As in Figs. 3b-g, but for weekly averaged daily Tmax forecast from the GEFS for the period of 1985-2010.

showed higher skill for maximum temperature (Figs. 4a,b). Despite improved correlations between GEFS and OBS in the nonmonsoon season, GEFS showed higher RMSE and bias in maximum temperature in the northern region, which can be attributed to the inability of the GEFS forecast to simulate maximum temperature in the higherelevation regions. Shah and Mishra (2014) reported that maximum temperature from the IMD has a high positive bias in the northern region as compared to the reanalysis products (ERA-Interim, MERRA, and CFSR), which may affect performance of the GEFS. The bias in the observed dataset of maximum temperature can be associated with the sparse gauge station network in northern India.

For mean minimum temperature, during the monsoon/ nonmonsoon seasons, the GEFS forecast showed lesser skill than that of mean maximum temperature for the period of 1985-2010 (Fig. 5). Median correlations 
between the OBS and GEFS forecasts ranged between 0.4 and 0.7 during the monsoon and nonmonsoon seasons, with more spatial variability (in correlations) in the northern and northeastern regions. Most of the regions showed a positive bias in minimum temperature during the monsoon season (Fig. 5e). On the other hand, a high negative bias was found in the northern region during the monsoon and nonmonsoon seasons. Overall, the results showed that the performance in simulating maximum and minimum temperatures is better in the nonmonsoon season, which is consistent with the simulation of precipitation. Higher skill in temperatures forecast during the nonmonsoon season can be explained on the basis of land-atmosphere interaction as well as the relationship between precipitation and temperature during the monsoon season. For instance, Tian and Martinez (2014) found lower skill in reference evapotranspiration in Florida during the summer season because of large convection activities that may not be well simulated by the GEFS model. Precipitation and air temperatures forecast from the GEFS consistently showed poor performance in the northern region, which can also be attributed to representation of aerosols (Gautam et al. 2009; Bollasina et al. 2011).

\section{b. Bias correction of the GEFS precipitation forecast}

We used linear scaling (Shah and Mishra 2015) for bias correction in weekly accumulated precipitation. Figure 6 shows mean absolute error (MAE) in the raw (interpolated to $0.25^{\circ}$ ) GEFS and average MAE from multifold trials (26 trials; 25 years for training and leaving 1 year for testing) for training and testing periods. Scale factors for the linear scaling were obtained for the training ( 25 years) period, and then their effectiveness was evaluated in the testing period (1 year; Fig. 6). The scaled precipitation forecast showed a significant improvement in the monsoon season for the training period in all of India and notably in the northeastern, Western Ghats, and central regions (Figs. 6b,e and Table 2). During the monsoon season in the testing period, the corrected forecast showed a reduction in MAE in foothills of the Himalayas, Western Ghats, south-central, and western regions (Figs. 6 c,f and Table 2 ). We find that the bias correction approach based on linear scaling substantially reduced the bias in precipitation in the monsoon and nonmonsoon seasons (Table 2).

Figure 4 in the supplemental material shows a comparison of areal mean weekly accumulated precipitation for each day of the forecast from the OBS and GEFS during the year 2010. The purpose of the comparison was to highlight the temporal nature of bias that varies in the selected regions. Our results demonstrated that the GEFS precipitation forecast has problems capturing precipitation totals because of ISV. The bias varies multiple times from negative to positive across all of India and in the selected regions because of the bias correction approaches that do not yield significant improvements (Fig. 4 in the supplemental material). Fan and Dool (2011) reported that in case of random bias within a season, the bias correction methods often provide little value. The timing error in prediction is evident in the selected regions during the monsoon season, which is associated with the ISV of precipitation during the monsoon season (see Figs. 1-3 in the supplemental material).

\section{c. Bias correction of daily maximum and minimum temperatures}

Figure 7 shows average MAE in weekly reforecasts of Tmax and Tmin from the GEFS for the monsoon and nonmonsoon seasons. The Q-Q mapping, which was used for bias correction of temperatures, resulted in improved results. Results showed that Tmax and Tmin from the GEFS have higher MAE in the Himalayan region during the monsoon and nonmonsoon seasons as compared to the OBS (Figs. 7a,d,g,j and Table 3). The error can be attributed to the quality of the observed temperatures in the region, as reported in Shah and Mishra (2014). We observed that the error in the GEFS Tmax forecast is lower in the southern peninsula (Figs. 7a,g). A relatively higher error can be seen in the GEFS forecast of Tmin during the monsoon and nonmonsoon seasons between 1985 and 2010 (Figs. 7d,j). Errors in Tmin were higher in the western regions of India, where differences in the GEFS and OBS were as high as $4^{\circ} \mathrm{C}$, which can be associated with the partitioning of the energy budget. However, further analysis is required to better understand the reasons behind the high bias in minimum temperature. The Q-Q mapping approach successfully improved bias across all of India during the training period of 25 years and testing period of 1 year, as revealed in multifold validation approach (Fig. 7 and Table 3).

\section{d. Influence of bias correction of the GEFS forcing on forecast skill of soil moisture and total runoff}

After correcting the bias in precipitation and maximum and minimum temperatures in the GEFS forecast, we evaluated the role of corrected and raw forcings on soil moisture and total runoff forecast using a single set of forcings (training period 1985-2009 and testing period 2010; see Figs. 4 and 5 in the supplemental material) from multifold validation. To evaluate the role of raw/corrected forcings, we designed three experiments using the retrospective simulated initial hydrologic condition (SIM) and forcings based on precipitation and 

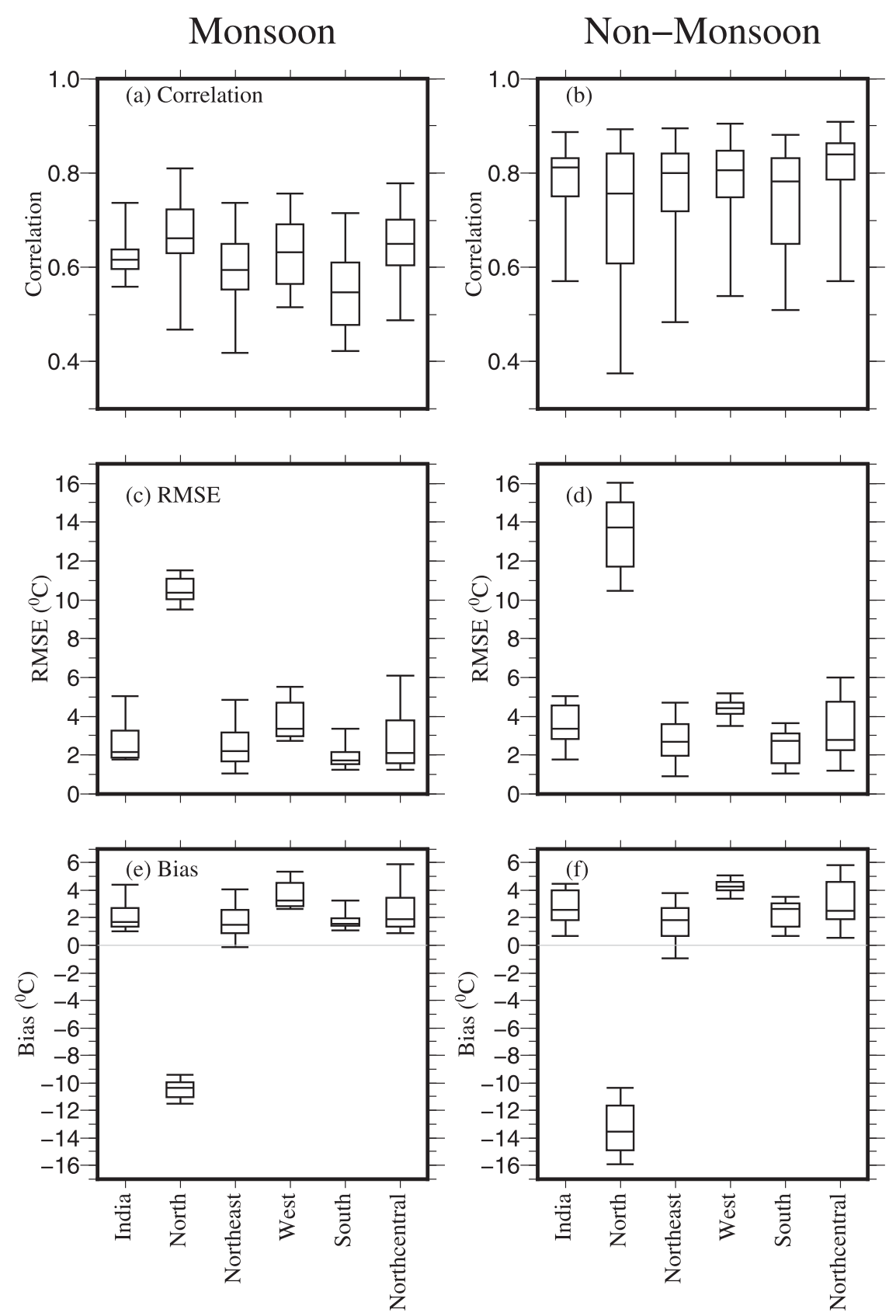

FIG. 5. As in Figs. 3b-g, but for weekly averaged daily Tmin.

temperature from observations (OBS from IMD), corrected GEFS (Co_GEFS), and raw GEFS (RAW) as shown in Table 1. We used simulated IHCs (SIM) and observed forcing (OBS from IMD) as the reference run, and performance of the other runs (SIM/RAW and SIM/ Co_GEFS) were evaluated against that.

Figure 7 in the supplemental material shows the comparison of mean (1985-2010) weekly top 10- and $60-\mathrm{cm}$ (root zone) soil moisture simulated using the SIM/OBS (retrospective simulated IHCs and observed forcing), SIM/RAW, and SIM/Co_GEFS on 15 February and 15 July. We considered 15 February and 15 July in each year to evaluate the forecast; however, skill can also be tested for other days of the forecast, which can provide a large sample size. The reference (SIM/OBS) soil moisture for the forecast initiated on 15 February showed high spatial variability with wetter soils in southern and northern India and low soil moisture in semiarid regions of western India (Fig. 7a in the supplemental material). Bias ( $\sim 5 \%)$ estimated using 


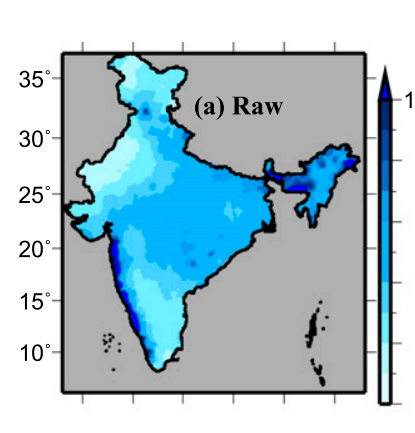

Monsoon

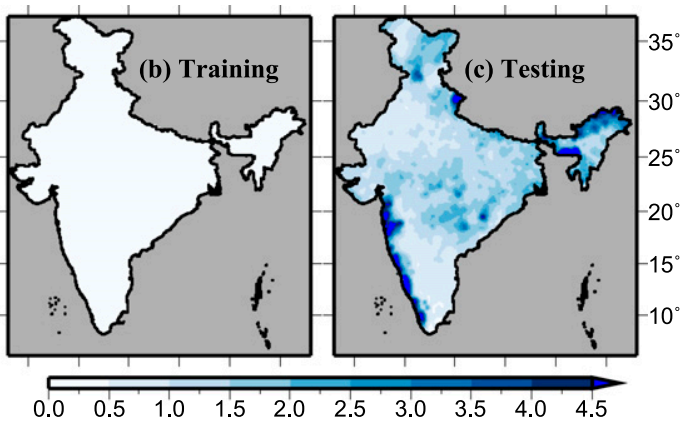

Non-Monsoon
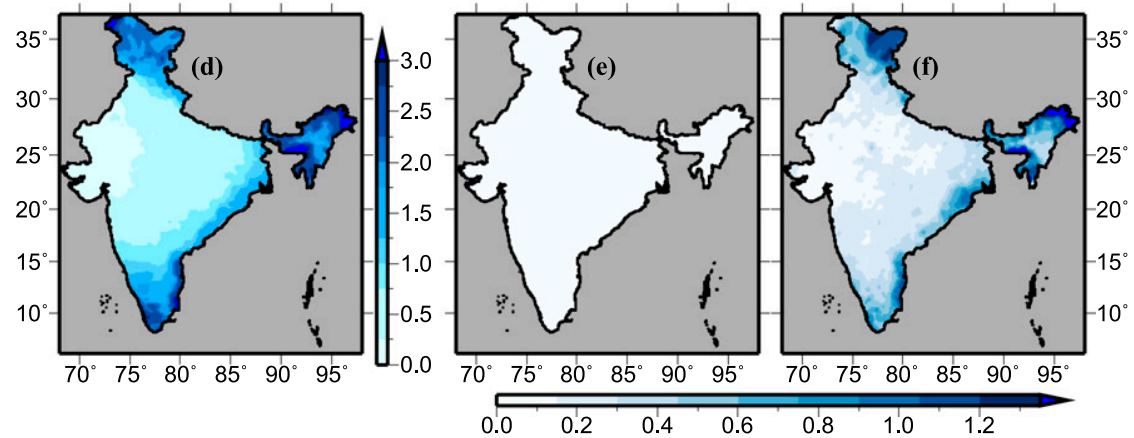

FIG. 6. MAE in precipitation predictions from GEFS before and after linear scaling. (a),(d) MAE in GEFS before linear scaling for the monsoon and nonmonsoon seasons. (b),(e) Mean of MAE from multifold trials (26 trials) during the training period (25 years) after linear scaling GEFS. (c),(f) As in (b) and (e), but for the testing period (1 year). forcings based on raw and corrected GEFS was negligible, which indicated the dominating role of IHCs in medium-range soil moisture forecast during the winter season. This result was expected because of high persistence in soil moisture (Figs. 7b,c in the supplemental material).

Experiments for the monsoon season that were run on 15 July of each year during the period of 1985-2010 confirmed the findings obtained from the experiments conducted for the winter season (Figs. 7d-f in the supplemental material). We find that the raw GEFS resulted in negative bias $(\sim 6 \%)$ in the Western Ghats, northern, and north-central regions, which was improved using the bias-corrected forcing from the GEFS. The bias in the top-layer soil moisture was improved with the corrected forcings from the GEFS. Our results demonstrate that corrected forcings may provide better estimates of changes in soil moisture anomalies at weekly scales.

Results for the root-zone soil moisture showed that bias correction of the GEFS forcing may not be very useful for hydrologic variables that have high

TABLE 2. Area-weighted mean (multifold trials) of MAE $\left(\mathrm{mm} \mathrm{day}^{-1}\right)$ in precipitation predictions from GEFS before and after the linear scaling was applied.

\begin{tabular}{|c|c|c|c|c|c|c|}
\hline \multirow[b]{2}{*}{ Region } & \multicolumn{3}{|c|}{ Monsoon } & \multicolumn{3}{|c|}{ Nonmonsoon } \\
\hline & Raw (1985-2010) & $\begin{array}{c}\text { Corrected } \\
\text { (training } \\
\text { period, } 25 \text { years) }\end{array}$ & $\begin{array}{c}\text { Corrected } \\
\text { (testing } \\
\text { period, } 1 \text { year) }\end{array}$ & Raw (1985-2010) & $\begin{array}{c}\text { Corrected } \\
\text { (training } \\
\text { period, } 25 \text { years })\end{array}$ & $\begin{array}{c}\text { Corrected } \\
\text { (testing } \\
\text { period, } 1 \text { year) }\end{array}$ \\
\hline India & 4.3 & 0 & 1.5 & 0.9 & 0 & 0.4 \\
\hline North & 3.0 & 0 & 1.6 & 1.7 & 0 & 0.6 \\
\hline Northeast & 6.0 & 0 & 2.1 & 1.5 & 0 & 0.6 \\
\hline West & 3.1 & 0 & 1.1 & 0.2 & 0 & 0.1 \\
\hline South & 4.4 & 0 & 1.5 & 1.2 & 0 & 0.4 \\
\hline North-central & 4.7 & 0 & 1.5 & 0.6 & 0 & 0.3 \\
\hline
\end{tabular}




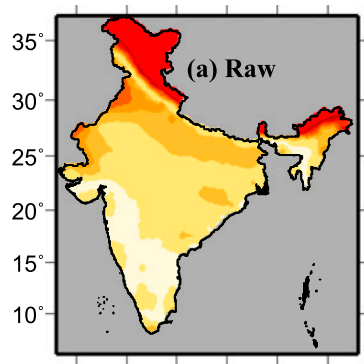

Tmax (Monsoon)
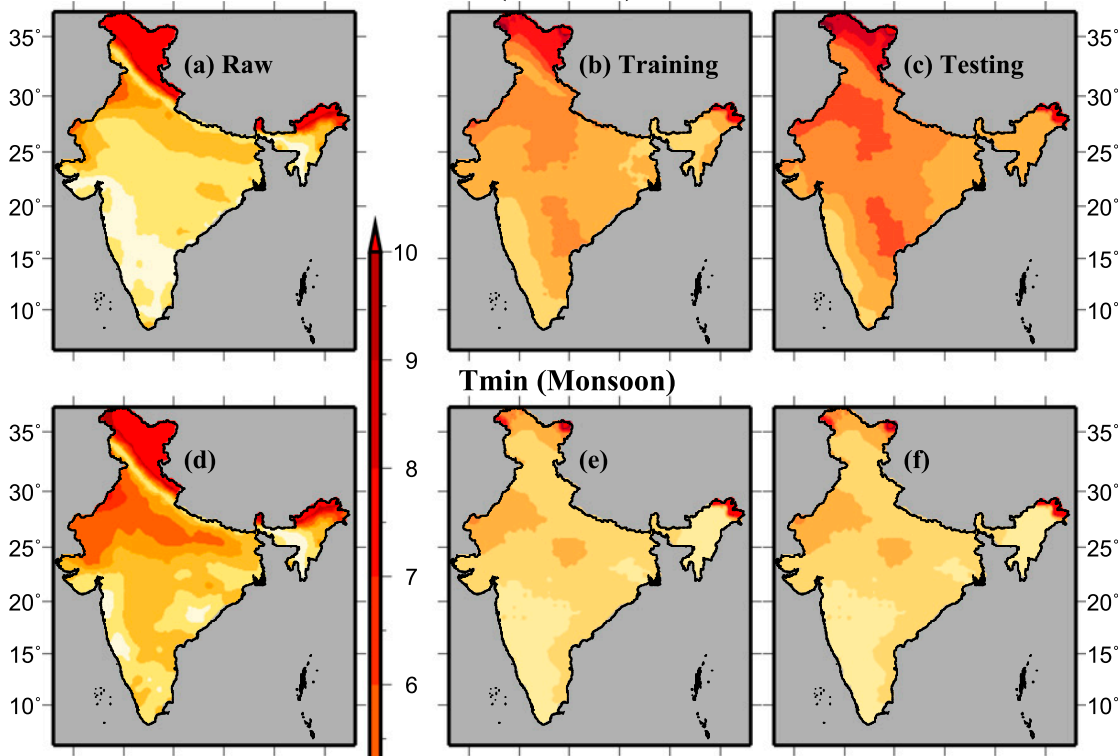

9

Tmin (Monsoon)
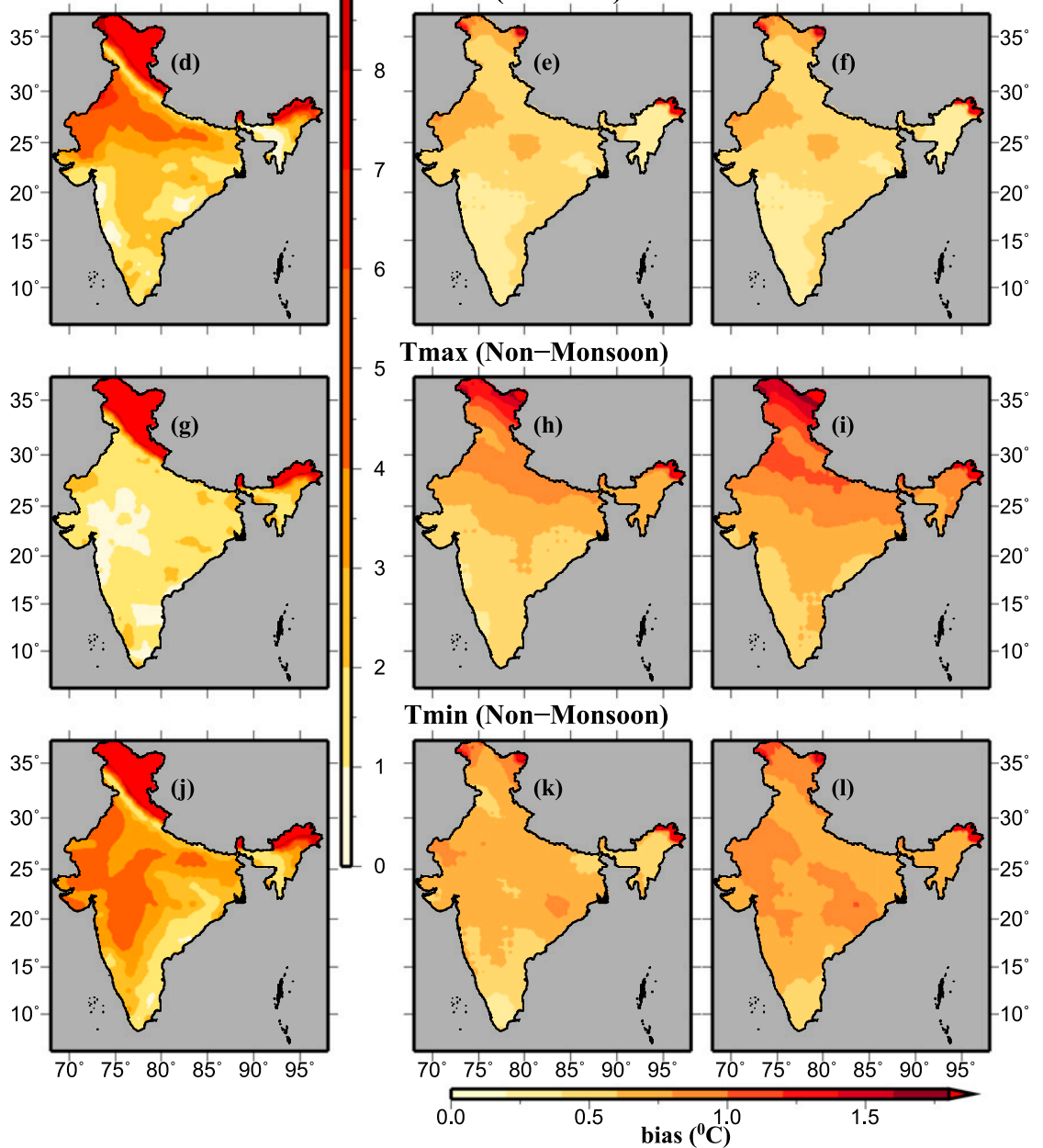

FIG. 7. MAE in Tmax and Tmin predictions from GEFS from OBS before and after Q-Q mapping. (a),(d),(g),(j) MAE in GEFS before Q-Q mapping monsoon and nonmonsoon seasons for Tmax and Tmin. (b),(e),(h),(k) Mean of MAE from multifold trials (26 trials) for the training period (25 years) in Q-Q mapped temperatures from GEFS. (c),(f),(i),(l) As in center panels, but for the testing period (1 year).

persistence in medium-range forecast. However, variables influenced by surface processes such as runoff can be affected by the quality of forcing for the shorter lead times. Therefore, we evaluated the role of corrected and raw forcings from the GEFS on the medium-range forecast of total runoff for the retrospective period of
1985-2010 (Fig. 8 in the supplemental material). For both of the days of forecast (15 February and 15 July), raw forcings from the GEFS with SIM IHCs resulted in a significant bias (up to $80 \%$ ) in total runoff simulations (Figs. 8b,c,e,f in the supplemental material). In both the seasons, SIM IHCs with the corrected GEFS forcing 
TABLE 3. Area-weighted mean (multifold trials) MAE $\left({ }^{\circ} \mathrm{C}\right)$ in Tmax and Tmin predictions from GEFS before and after Q-Q mapping was applied.

\begin{tabular}{|c|c|c|c|c|c|c|}
\hline \multirow[b]{2}{*}{ Region } & \multicolumn{3}{|c|}{ Tmax } & \multicolumn{3}{|c|}{ Tmin } \\
\hline & Raw (1985-2010) & $\begin{array}{c}\text { Corrected } \\
\text { (training } \\
\text { period, } 25 \text { years) }\end{array}$ & $\begin{array}{c}\text { Corrected } \\
\text { (testing, } 1 \text { year) }\end{array}$ & Raw (1985-2010) & $\begin{array}{c}\text { Corrected } \\
\text { (training } \\
\text { period, } 25 \text { years) }\end{array}$ & $\begin{array}{c}\text { Corrected } \\
\text { (testing, } 1 \text { year) }\end{array}$ \\
\hline \multicolumn{7}{|l|}{ Monsoon } \\
\hline India & 3.1 & 0.8 & 0.9 & 3.7 & 0.5 & 0.6 \\
\hline North & 11.7 & 1.1 & 1.2 & 9.6 & 0.6 & 0.7 \\
\hline Northeast & 3.0 & 0.7 & 0.8 & 3.1 & 0.5 & 0.6 \\
\hline West & 1.8 & 0.8 & 0.9 & 3.7 & 0.5 & 0.6 \\
\hline South & 1.1 & 0.7 & 0.8 & 1.8 & 0.4 & 0.4 \\
\hline North-central & 2.0 & 0.8 & 0.9 & 2.9 & 0.5 & 0.6 \\
\hline \multicolumn{7}{|l|}{ Nonmonsoon } \\
\hline India & 3.4 & 0.7 & 0.8 & 4.6 & 0.7 & 0.8 \\
\hline North & 15.0 & 1.1 & 1.3 & 12.8 & 0.7 & 0.9 \\
\hline Northeast & 3.2 & 0.8 & 0.9 & 3.7 & 07 & 0.9 \\
\hline West & 1.3 & 0.6 & 0.8 & 4.3 & 0.7 & 0.8 \\
\hline South & 1.3 & 0.5 & 0.6 & 2.5 & 0.6 & 0.7 \\
\hline North-central & 1.8 & 0.7 & 0.8 & 3.4 & 0.7 & 0.8 \\
\hline
\end{tabular}

resulted in the best simulations of total runoff. In comparison to soil moisture simulations, the role of IHCs is less dominating for total runoff, which can be attributed to the relative influence of persistence of both the hydrologic variables. We expect that the medium-range forecast of total runoff may be more influenced by the GEFS forcing because of lower persistence than soil moisture. For improved hydrologic forecast at shorter lead times, the availability of OBS initial conditions will be desirable (Table 4). Satellite-based observations can be used in real time in countries like India (Shah and Mishra 2015); however, there are known biases in forcings from satellite or reanalysis products (Shah and Mishra 2014).

\section{e. Evaluation of GEFS forecast against observed streamflow, soil moisture, and drought}

\section{1) StREAMFLOW}

The VIC was calibrated and evaluated at $0.25^{\circ}$ spatial resolution against the observed soil moisture and streamflow in the previous studies (Mishra et al. 2014; Shah and Mishra 2015). We evaluated the effectiveness of the VIC to simulate monthly and daily streamflow at Garudeshwar station located in the Narmada River basin in western India (Fig. 8). It can be noted from the results that the VIC successfully reproduced the observed monthly and daily streamflow with NashSutcliffe efficiency (NSE) values of 0.70 and 0.68 , respectively, for the period of 1973-2010 (Figs. 8a-c). We evaluated forecast skill of the GEFS for weekly streamflow simulations at the Garudeshwar station in the Narmada River basin for 1985-2010 (Fig. 8d). Since observed streamflow data for a few years were missing during the period of 1985-2010, we limit our comparisons for the years with available observed data. We compared the VIC simulated flow using the retrospective simulated initial conditions with observed (SIM/ OBS), raw (SIM/RAW), and corrected (SIM/Co_ GEFS) forcings (Fig. 8d). Weekly mean streamflow was compared for the forecast initiated on 15 July of each year for which observed streamflow data were available. We notice that the GEFS can provide reasonable estimates of weekly streamflow at the gauge station in a few years; however, results showed a large bias in some years (e.g., 1993). The bias in the GEFS forecast of daily streamflow can be attributed to bias in forcings during these years. In general, we find no significant improvement in the simulation of streamflow using the corrected and uncorrected GEFS forcing, which may be related to the role of soil moisture persistence in the basin.

TABLE 4. Area-weighted (for India) MAE in soil moisture and total runoff simulated using OBS IHCs and uncorrected and corrected forcings from the GEFS during the period of 1985-2010.

\begin{tabular}{lcccc}
\hline \hline & \multicolumn{2}{c}{ IHC } & & \multicolumn{2}{c}{$\begin{array}{c}\text { Retrospective } \\
\text { simulations }\end{array}$} \\
\cline { 2 - 2 } \cline { 5 - 5 } \cline { 5 - 5 } & Forecast forcing & & RAW & Co_GEFS \\
\hline Soil moisture (mm) & $15 \mathrm{Feb}$ & & 0.74 & 0.27 \\
Top soil moisture & $15 \mathrm{Jul}$ & & 2.73 & 0.85 \\
$\quad$ fraction) & $15 \mathrm{Feb}$ & & 0.40 & 0.25 \\
Runoff $(\mathrm{mm})$ & & & & \\
& $15 \mathrm{Jul}$ & & 0.95 & 0.52 \\
& $15 \mathrm{Feb}$ & & 0.08 & 0.02 \\
& $15 \mathrm{Jul}$ & & 1.15 & 0.41 \\
\hline
\end{tabular}


Garud (Narmada)
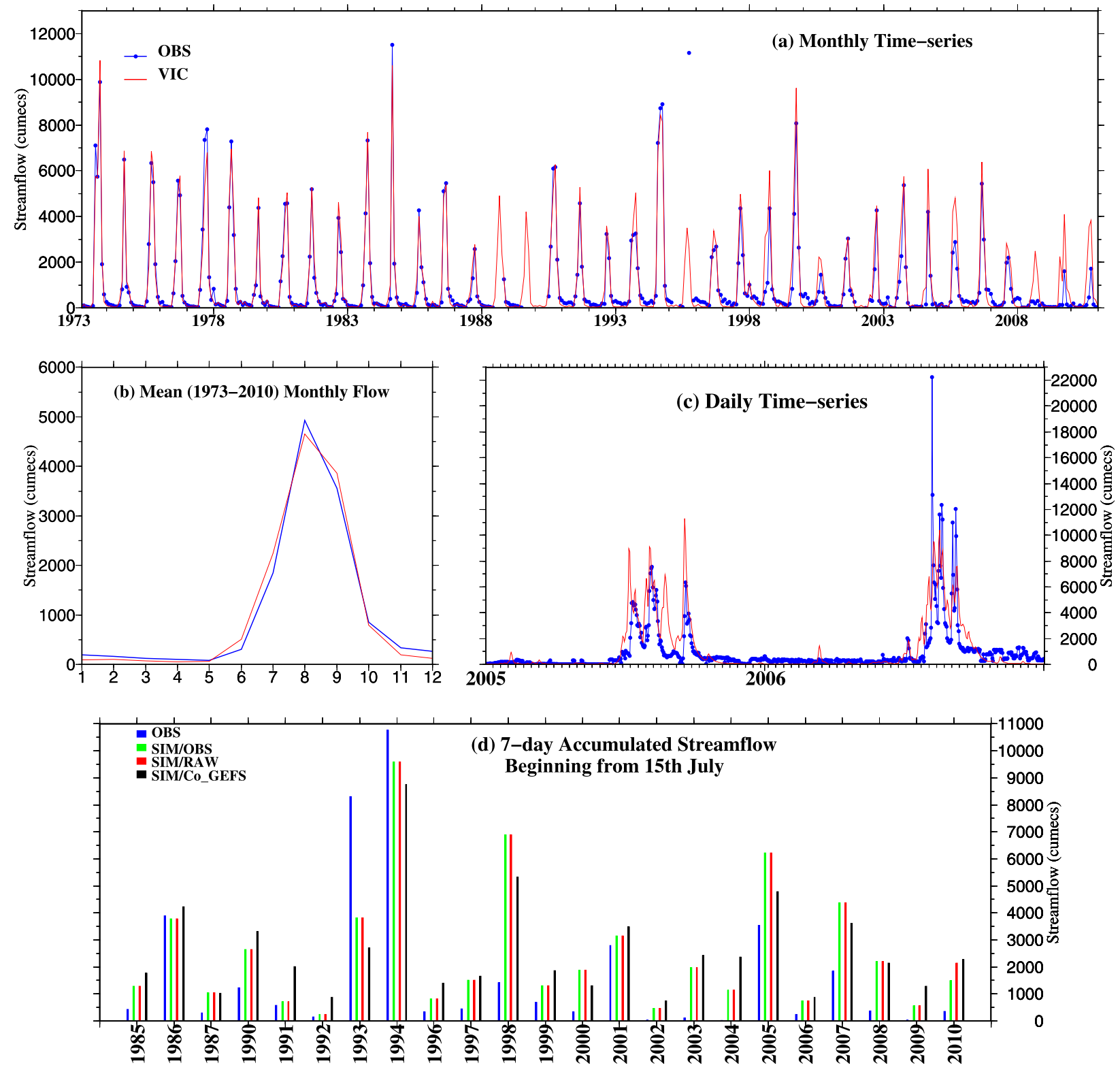

FIG. 8. Evaluation of streamflow simulated using the VIC and OBS forcing at Garud gauge station in the Narmada basin against observed streamflow. (a) Observed and simulated monthly during the period 1973-2010, (b) seasonal cycle of observed and simulated monthly streamflow for the period 1973-2010, (c) observed and simulated daily streamflow during 2005-06, and (d) comparison of streamflow simulated using VIC and OBS forcing and forcing based on uncorrected and corrected GEFS with OBS streamflow for weekly predictions initiated on 15 Jul.

\section{2) SOIL MOISTURE}

The VIC-simulated soil moisture for India was evaluated against satellite-based observations obtained from the ESACCI in Mishra et al. (2014). Moreover, Mishra et al. (2014) evaluated the VIC-simulated soil moisture against the in situ data from the International Soil Moisture Network (ISMN) at the station located in the Indian Institute of Technology (IIT) Kanpur. We evaluated the soil moisture forecast obtained using the retrospective simulated initial conditions and corrected and raw forcing from the GEFS against the satellite soil moisture. Figure 9 shows the correlation between simulated soil moisture obtained using the corrected/ raw GEFS forcings and satellite-based soil moisture for the forecast initiated on 15 February and 15 July. Correlations for the winter season are similar for soil moisture obtained using the corrected and raw GEFS 


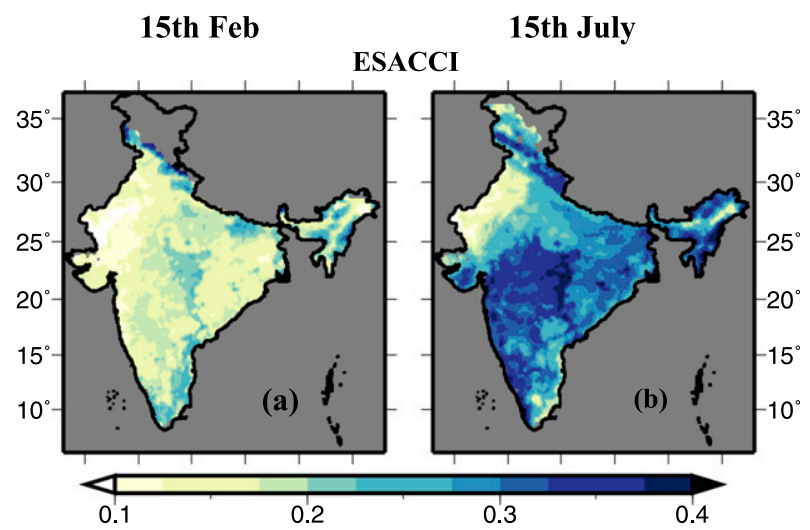

Correlation (SIM/ RAW)
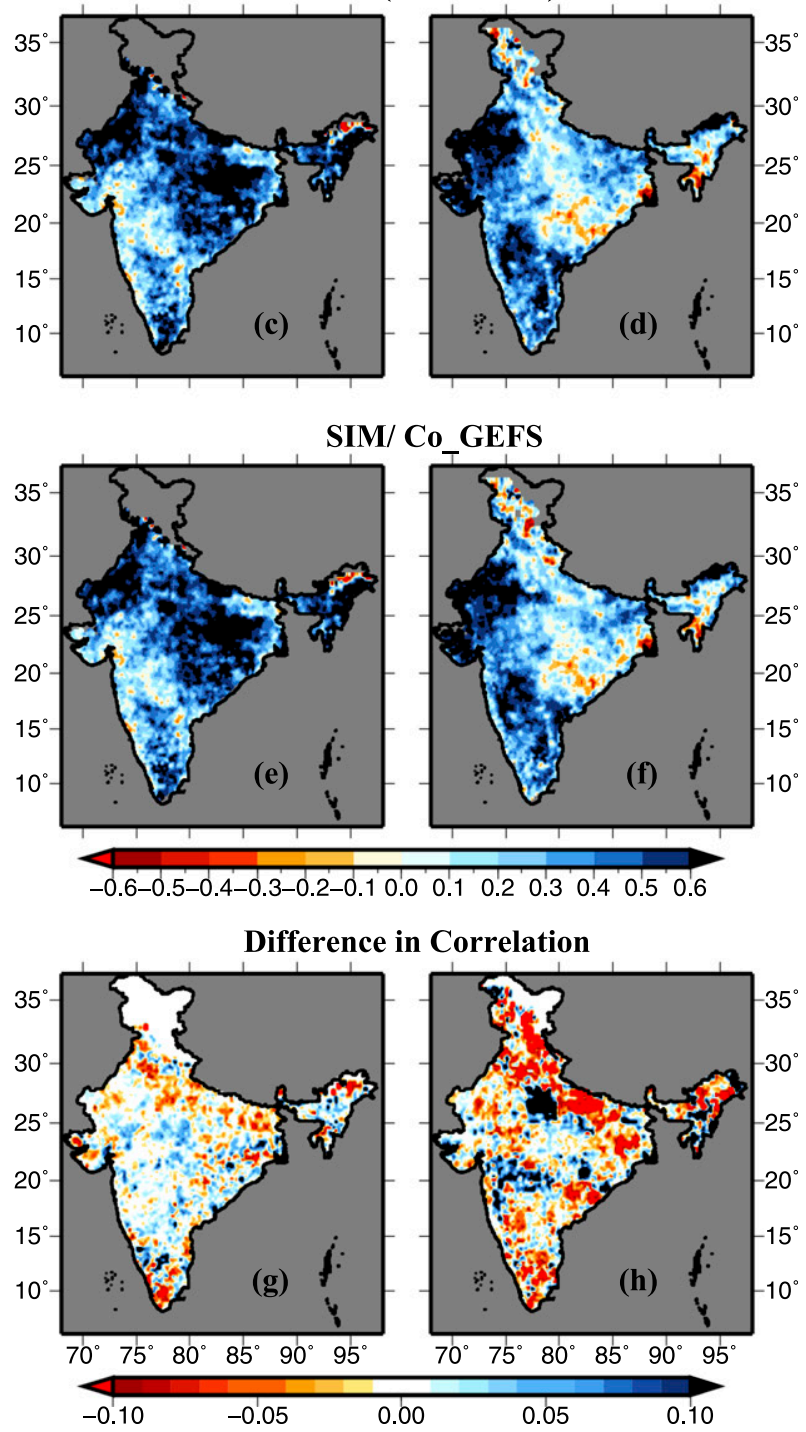

FIG. 9. Correlation (1985-2010) of predicted top 10-cm soil moisture with OBS soil moisture from ESACCI. (a),(b) Satellite-driven mean surface soil moisture for 15-22 Feb and 15-22 Jul for the period of 19852010. (c),(d) Correlation between satellite-driven soil moisture and predicted soil moisture using the raw GEFS forecast and observed initial conditions for the forecast initiated on $15 \mathrm{Feb}$ and $15 \mathrm{Jul}$, respectively, of each year. (e),(f) As in (c) and (d), but for the corrected GEFS forcing. forcing, which once again highlights the role of IHCs. In the monsoon, improvement was noticed in only a few regions due to bias correction of the GEFS forcing (Fig. 9). Results also suggested that forecast skill is better for soil moisture simulations during the nonmonsoon season than the monsoon season (Fig. 9). Higher correlations during the winter season are promising, as soil moisture forecast can be valuable for irrigation water management.

\section{3) Drought}

Figure 9 in the supplemental material shows probability of detection (POD; ratio of predicted drought events against observed total drought events) obtained using soil moisture droughts [based on standardized soil moisture index (SSI) $<-0.5$; see Shah and Mishra (2015) for more details on soil moisture index] from the GEFS corrected and raw forcings and retrospective simulated initial conditions with satellite-based drought estimations based on DSI ( $\mathrm{Mu}$ et al. 2013). Results show higher POD for the nonmonsoon season than for the monsoon season (Fig. 9 in the supplemental material). Moreover, results showed no significant difference in POD in the corrected and raw forcings from the GEFS, except in the north for the forecast initiated on 15 February (Figs. 9e,g in the supplemental material) and in the west during the monsoon season (Figs. 9f,h in the supplemental material). Based on POD and false alarm ratio (FAR; Fig. 10 in the supplemental material; see Wilks 2006), we find that predictability of drought is lower in the south with higher FAR but higher in the western regions with lower FAR and higher POD.

Figure 10 shows predicted anomalies of precipitation, air temperature, and surface and root-zone soil moisture for the period of 15-22 July 2002. Comparison of the predicted (based on the corrected GEFS) and observed anomalies highlights the utility of medium-range forecasts for the agriculture and water community. For instance, weekly precipitation and soil moisture deficit and positive temperature anomalies were predicted well in northern India, which can be used for planning and decision-making. Moreover, we evaluated the weekly drought forecast against satellitebased drought estimates (i.e., DSI; Mu et al. 2013) for 15 July as the day of forecast (Fig. 11) for years 2002, 2004, 2005, and 2009. To make SSI consistent with DSI (climatological period 2000-11), we used the period 2000-10 as the climatological period for SSI. Our results show that severity of drought increases from the week ending on 14 July (Fig. 11a) to 22 July 2002 (Fig. 11b), which was predicted well by both corrected and raw forcings (Figs. 11c,d) and was comparable to 

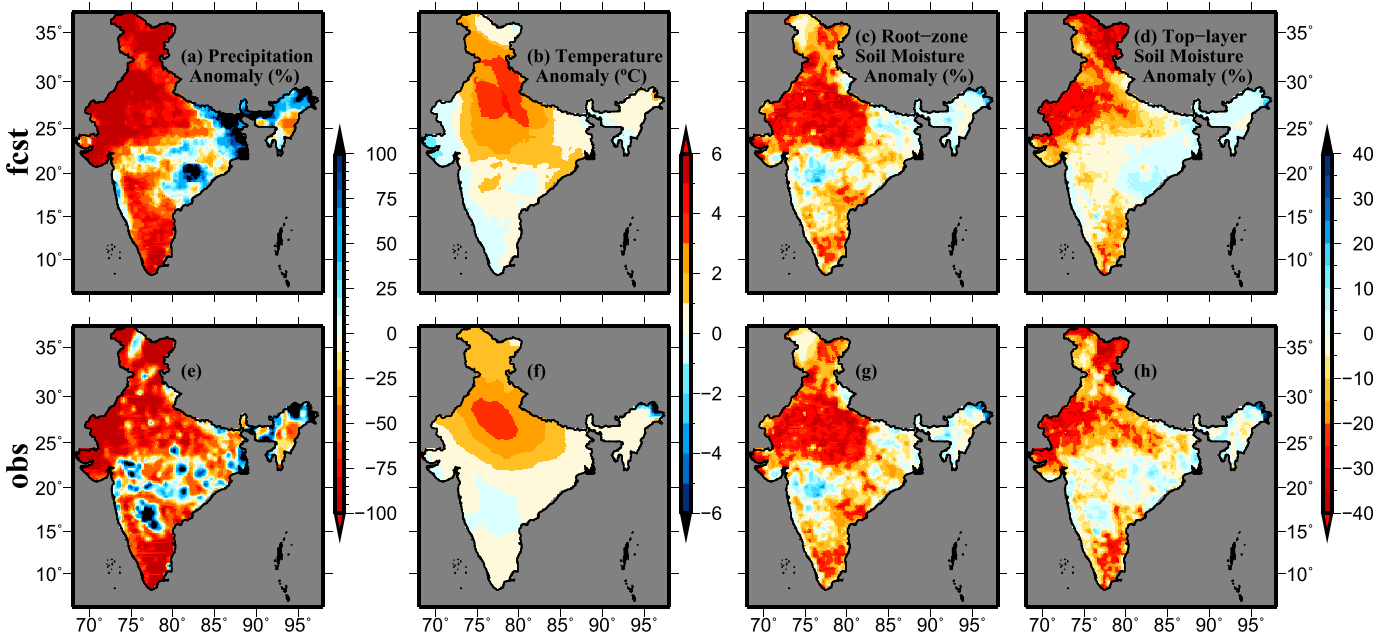

FIG. 10. Comparison of observed and predicted anomalies of precipitation, air temperature, and surface and root-zone soil moisture using the corrected GEFS forcing and VIC during 15-22 Jul 2002.

DSI (Fig. 11e). The weekly drought prediction system was able to reproduce the change in drought in July 2002. The effectiveness of the medium-range forecast of drought can be found on the basis of change in anomalies on the weekly basis. Similarly, we find that the medium-range drought forecast captured the increase in severity of drought in the western region for the week ending on 22 July 2004 (Figs. 11f-j). Similar results were also obtained for other forecast dates (Fig. 11). These results highlight the importance of the prediction of change in drought at the weekly scale, which can be valuable for farmers and water managers.

\section{Conclusions}

Based on our findings, we conclude the following:

1) Medium-range forecast skill of the GEFS varies in seasons as well as in different regions in India. For instance, forecast skill evaluated using Spearman rank correlation, RMSE, and bias showed that the GEFS performs better in the nonmonsoon season than in the monsoon season for all the selected variables (e.g., precipitation, Tmax, and Tmin). For both maximum and minimum temperatures, a warm bias was observed in the GEFS forecast in the monsoon and nonmonsoon seasons. Overall, for precipitation and air temperatures, forecast skill was lower in the northern, northeastern, and core monsoon regions, which can be attributed to the ability of the GEFS to simulate intraseasonal variability in the monsoon season.

2) After the evaluation of the forecast skill in precipitation and maximum and minimum temperatures, we postprocessed the GEFS forecast for hydrologic applications. We evaluated the linear scaling and Q-Q mapping for bias correction of precipitation and air temperatures, respectively, for the period of 19852010. Our results showed that the Q-Q mapping approach can be successfully used to correct bias in maximum and minimum temperatures. We used the linear scaling to correct bias in precipitation forecast. The linear scaling approach effectively improved bias in precipitation forecast in both monsoon and nonmonsoon seasons.

3) We evaluated the influence of bias correction of precipitation and temperatures from the GEFS on forecast skill of soil moisture, runoff, and streamflow. Our results showed that, because of high persistence, medium-range soil moisture forecast is significantly affected by the initial hydrologic conditions. For instance, initial hydrologic conditions have higher skill in root-zone soil moisture forecast, and the best forecast skill was obtained using the retrospective simulated initial condition. For total runoff and surface soil moisture simulations, the role of initial hydrologic condition was lower than that in root-zone soil moisture, which highlighted that both atmospheric forcing and initial condition play an important role in total runoff and surface soil moisture forecast. Overall, observed initial hydrologic conditions and corrected GEFS forcing resulted in the most improved forecast for the total runoff. In a developing country like India, availability of gauge-based observations of soil moisture, precipitation, and temperatures is limited in near-real time. Satellite-based precipitation and hydrologic models can be used to simulate soil moisture, which can provide initial state 


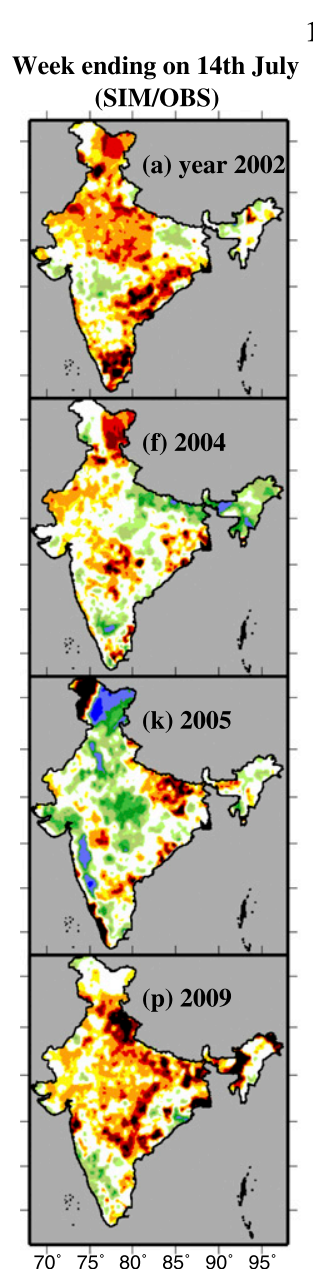

1-week Standardized Soil Moisture Index

\section{Week ending on 22nd July}

(SIM/OBS)
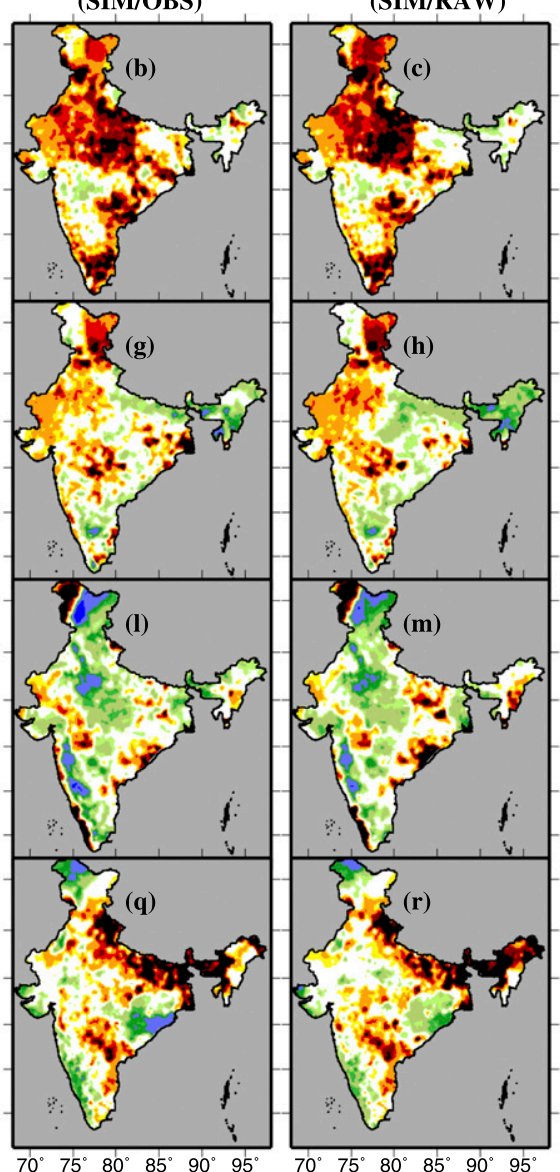

DSI
(SIM/Co_GEFS)

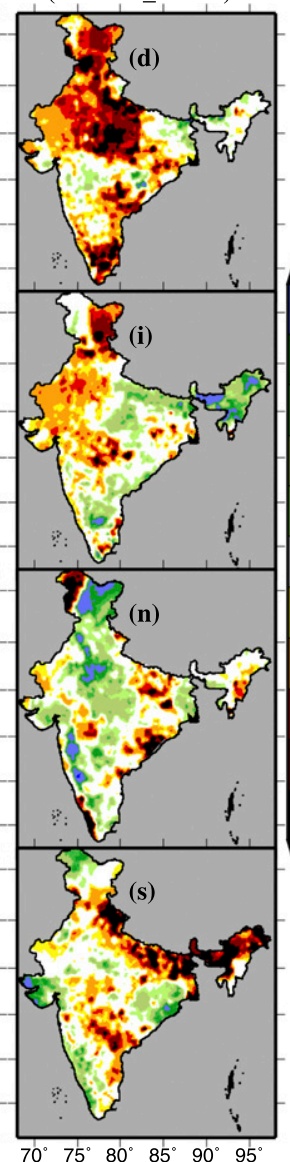

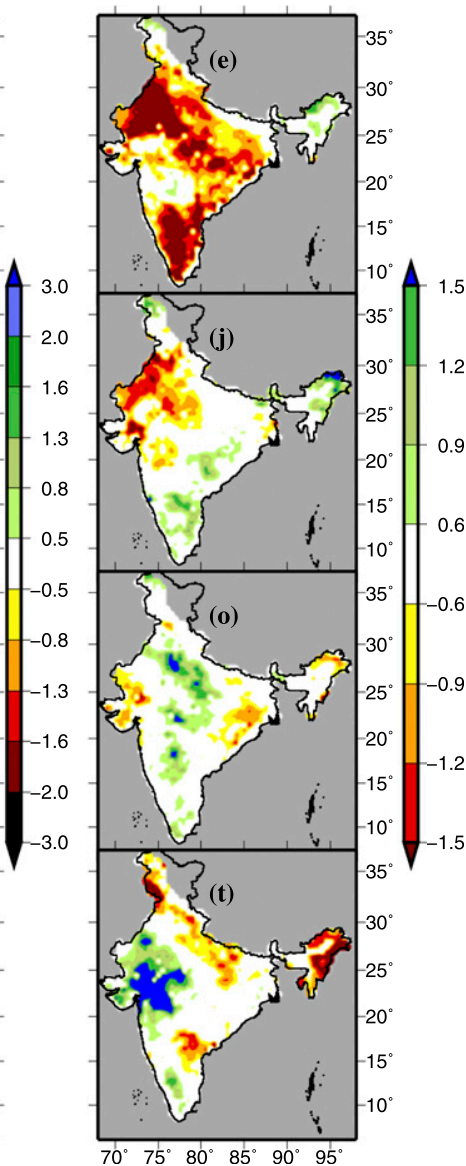

FIG. 11. Comparison of short-term drought forecast from the corrected and uncorrected GEFS forcing against the observed drought based on satellite and IMD forcing. (a),(f),(k),(p) One-week SSI estimated based on soil moisture simulated using OBS/OBS for week ending on 14 Jul 2002, 2004, 2005, and 2009, respectively. (b),(g),(l),(q) As in (a),(f),(k),(p), but for week ending on 22 Jul. (c),(h),(m),(r) One-week SSI estimated soil moisture simulated using OBS/Un_GEFS for week ending on 22 Jul for the selected years. (d),(i),(n),(s) As in (c),(h),(m),(r), but for the OBS/Co_GEFS. (e),(j),(o),(t) Mean of DSI for 8-day composite ending on 20 and 27 Jul for the selected periods.

for hydrologic prediction (Shah and Mishra 2015; Sheffield et al. 2014; Wu et al. 2014).

4) Weekly forecasts of surface soil moisture, streamflow, and drought were evaluated against the observed and satellite datasets for the winter and monsoon seasons. Results suggested better forecast skill for the nonmonsoon season than that of the monsoon season. Moreover, weekly mean streamflow showed reasonable forecast skill for most of the years; however, in a few years random bias was found in the forecast. Changes in weekly droughts and anomalies of precipitation, air temperature, and soil moisture were predicted well by the GEFS-based medium-range forecast system. Future work will be required to use data assimilation and to explore more robust techniques to correct the GEFS forcing and observed initial conditions.
Acknowledgments. The authors acknowledge data availability from the GEFS reforecast. Moreover, funding from the Government of India Ministry of Human Resource Development (MHRD) and the Varahmihir Ministry of Earth Sciences is greatly appreciated. The work is undertaken as part of the Information Technology Research Academy (ITRA), Media Lab Asia project entitled "Measurement to Management (M2M): Improved Water Use Efficiency and Agricultural Productivity through Experimental Sensor Network." The authors appreciate valuable comments on the previous version from Upmanu Lall, Columbia University. The authors appreciate constructive comments and suggestions from the Editor (Andrew Wood) that helped in improving the manuscript. 


\section{REFERENCES}

Abraham, Z., P. Tan, Perdinan, J. Winkler, S. Zhong, and M. Liszewska, 2013: Position preserving multi-output prediction. Machine Learning and Knowledge Discovery in Databases, H. Blockeel et al., Eds., Lecture Notes in Computer Science, Vol. 8189, 320-335, doi:10.1007/978-3-642-40991-2_21.

Ashrit, R., and Coauthors, 2013: Performance of Global Ensemble Forecast System (GEFS) during monsoon 2012. Rep. NMRF/ RR/1/2013, NCMRWF, 27 pp. [Available online at http://www. ncmrwf.gov.in/GEFS_Report_Final.pdf.]

Bollasina, M. A., Y. Ming, and V. Ramaswamy, 2011: Anthropogenic aerosols and the weakening of the South Asian summer monsoon. Science, 334, 502, doi:10.1126/science.1204994.

Chaudhari, H., S. Pokhrel, S. Mohanty, and S. Saha, 2013: Seasonal prediction of Indian summer monsoon in NCEP coupled and uncoupled model. Theor. Appl. Climatol., 114, 459-477, doi:10.1007/s00704-013-0854-8.

Cherkauer, K. A., L. C. Bowling, and D. P. Lettenmaier, 2003: Variable Infiltration Capacity cold land process model updates. Global Planet. Change, 38, 151-159, doi:10.1016/ S0921-8181(03)00025-0.

Dee, D., and Coauthors, 2011: The ERA-Interim reanalysis: Configuration and performance of the data assimilation system. Quart. J. Roy. Meteor. Soc., 137, 553-597, doi:10.1002/qj.828.

Dorigo, W., R. Jeu, D. Chung, R. Parinussa, Y. Liu, W. Wagner, and D. Fernández-Prieto, 2012: Evaluating global trends (19882010) in harmonized multi-satellite surface soil moisture. Geophys. Res. Lett., 39, L18405, doi:10.1029/2012GL052988.

Durai, V., and S. Bhowmik, 2014: Prediction of Indian summer monsoon in short to medium range time scale with high resolution Global Forecast System (GFS) T574 and T382. Climate Dyn., 42, 1527-1551, doi:10.1007/s00382-013-1895-5.

Fan, Y., and V. Dool, 2011: Bias correction and forecast skill of NCEP GFS Ensemble week-1 and week-2 precipitation, 2-m surface air temperature, and soil moisture forecasts. Wea. Forecasting, 26, 355-370, doi:10.1175/WAF-D-10-05028.1.

Gao, H., and Coauthors, 2009: Water budget record from Variable Infiltration Capacity (VIC) model. Algorithm Theoretical Basis Doc., version 1.2, 56 pp. [Available online at http://www.hydro. washington.edu/SurfaceWaterGroup/Publications/Water_Cycle_ MEaSUREs_ATBD_VICmodel_submit.doc.]

Gautam, R., N. C. Hsu, K.-M. Lau, and M. Kafatos, 2009: Aerosol and rainfall variability over the Indian monsoon region: Distributions, trends and coupling. Ann. Geophys., 27, 3691-3703, doi:10.5194/angeo-27-3691-2009.

Gutiérrez, J. M., A. S. Cofiño, R. Cano, and M. A. Rodríguez, 2004: Clustering methods for statistical downscaling in short-range weather forecasts. Mon. Wea. Rev., 132, 2169-2183, doi:10.1175/1520-0493(2004)132<2169:CMFSDI >2.0.CO;2.

Hamill, T. M., G. T. Bates, J. S. Whitaker, D. R. Murray, M. Fiorino, T. J. Galarneau Jr., Y. Zhu, and W. Lapenta, 2013: NOAA's second-generation global medium-range ensemble forecast dataset. Bull. Amer. Meteor. Soc., 94, 1553-1565, doi:10.1175/BAMS-D-12-00014.1.

Hansen, M. C., R. S. DeFries, J. R. Townshend, and R. Sohlberg, 2000: Global land cover classification at $1 \mathrm{~km}$ spatial resolution using a classification tree approach. Int. J. Remote Sens., 21, 1331-1364, doi:10.1080/014311600210209.

Kessler, W. S., 2001: EOF representation of the Madden-Julian oscillation and its connection with ENSO. J. Climate, 14, 3055-3061, doi:10.1175/1520-0442(2001)014<3055: EROTMJ $>2.0 . \mathrm{CO} ; 2$.
Krishnamurthy, V., and J. Shukla, 2008: Seasonal persistence and propagation of intraseasonal patterns over the Indian monsoon region. Climate Dyn., 30, 353-369, doi:10.1007/s00382-007-0300-7.

Kumar, A., P. Maini, L. Rathore, and S. Singh, 2000: An operational medium range local weather forecasting system developed in India. Int. J. Climatol., 20, 73-87, doi:10.1002/ (SICI)1097-0088(200001)20:1<73::AID-JOC454>3.0.CO;2-Q.

Liang, X., 1994: A two-layer variable infiltration capacity land surface representation for general circulation models. Ph.D. thesis, University of Washington, $208 \mathrm{pp}$.

— D. P. Lettenmaier, and E. F. Wood, 1996: One-dimensional statistical dynamic representation of subgrid spatial variability of precipitation in the two-layer Variable Infiltration Capacity model. J. Geophys. Res., 101, 21 403-21 421, doi:10.1029/ 96JD01448.

Madadgar, S., H. Moradkhani, and D. Garen, 2014: Towards improved post-processing of hydrologic forecast ensembles. Hydrol. Processes, 28, 104-122, doi:10.1002/hyp.9562.

Maurer, E. P., A. W. Wood, J. C. Adam, D. P. Lettenmaier, and B. Nijssen, 2002: A long-term hydrologically based dataset of land surface fluxes and states for the conterminous United States. J. Climate, 15, 3237-3251, doi:10.1175/1520-0442(2002)015<3237: ALTHBD $>2.0 . C O ; 2$.

Mishra, V., 2015: Climatic uncertainty in Himalayan water towers. J. Geophys. Res. Atmos., 120, 2689-2705, doi:10.1002/ 2014JD022650.

_ R. Shah, and B. Thrasher, 2014: Soil moisture droughts under the retrospective and projected climate in India. J. Hydrometeor., 15, 2267-2292, doi:10.1175/JHM-D-13-0177.1. , B. V. Smoliak, D. P. Lettenmaier, and J. M. Wallace, 2012: A prominent pattern of year-to-year variability in Indian summer monsoon rainfall. Proc. Natl. Acad. Sci. USA, 109, 7213-7217, doi:10.1073/pnas.1119150109.

Misra, V., P. A. Dirmeyer, and B. P. Kirtman, 2003: Dynamic downscaling of seasonal simulations over South America. J. Climate, 16, 103-117, doi:10.1175/1520-0442(2003)016<0103: DDOSSO $>2.0 . \mathrm{CO} ; 2$.

Mu, Q., M. Zhao, J. S. Kimball, N. G. McDowell, and S. W. Running, 2013: A remotely sensed global terrestrial drought severity index. Bull. Amer. Meteor. Soc., 94, 83-98, doi:10.1175/ BAMS-D-11-00213.1.

Narsimhan, B., and R. Srinivasan, 2005: Development and evaluation of soil moisture deficit index (SMDI) and evapotranspiration deficit index (ETDI) for agricultural drought monitoring. Agric. For. Meteor., 133, 69-88, doi:10.1016/j.agrformet.2005.07.012.

Nijssen, B., R. Schnur, and D. P. Lettenmaier, 2001: Global retrospective estimation of soil moisture using the Variable Infiltration Capacity land surface model, 1980-93. J. Climate, 14, 1790-1808, doi:10.1175/1520-0442(2001)014<1790:GREOSM>2.0.CO;2.

Pai, D. S., L. Sridhar, M. R. Badwaik, and M. Rajeevan, 2014: Analysis of the daily rainfall events over India using a new long period $(1901-2010)$ high resolution $\left(0.25^{\circ} \times 0.25^{\circ}\right)$ gridded rainfall dataset. Climate Dyn., 45, 755-776, doi:10.1007/s00382-014-2307-1.

Palmer, W. C., 1968: Keeping track of crop moisture conditions, nationwide: The new crop moisture index. Weatherwise, 21, 156-161, doi:10.1080/00431672.1968.9932814.

Rajeevan, M., S. Gadgil, and J. Bhate, 2010: Active and break spells of the Indian summer monsoon. J. Earth Syst. Sci., 119, 229-247, doi:10.1007/s12040-010-0019-4.

Rathore, L., and P. Maini, 2008: Economic impact assessment of agro-meteorological advisory service of NCMRWF. Rep. NMRF/PR/1/2008, NCMRWF, 116 pp. [Available online at http://www.ncmrwf.gov.in/NCMRWF_REPORT.pdf.] 
Saha, S., and Coauthors, 2010: The NCEP Climate Forecast System Reanalysis. Bull. Amer. Meteor. Soc., 91, 1015-1057, doi:10.1175/2010BAMS3001.1.

Saseendran, S., S. Singh, L. Rathore, and S. Das, 2002: Characterization of weekly cumulative rainfall forecasts over meteorological subdivisions of India using a GCM. Wea. Forecasting, 17, 832-844, doi:10.1175/1520-0434(2002)017<0832: COWCRF $>2.0 . \mathrm{CO} ; 2$.

Shah, R., and V. Mishra, 2014: Evaluation of the reanalysis products for the monsoon season droughts in India. J. Hydrometeor., 15, 1575-1591, doi:10.1175/JHM-D-13-0103.1.

$\longrightarrow$, and — 2015: Development of an experimental near-realtime drought monitor for India. J. Hydrometeor., 16, 327-345, doi:10.1175/JHM-D-14-0041.1.

Sheffield, J., G. Goteti, and E. F. Wood, 2006: Development of a 50-year high resolution global dataset of meteorological forcings for land surface modeling. J. Climate, 19, 3088-3111, doi:10.1175/JCLI3790.1.

— system for sub-Saharan Africa water resources and food security. Bull. Amer. Meteor. Soc., 95, 861-882, doi:10.1175/ BAMS-D-12-00124.1.

Shukla, S., and D. P. Lettenmaier, 2011: Seasonal hydrologic prediction in the United States: Understanding the role of initial hydrologic conditions and seasonal climate forecast skill. Hydrol. Earth Syst. Sci., 15, 3529-3538, doi:10.5194/hess-15-3529-2011.

_, N. Voisin, and D. Lettenmaier, 2012: Value of medium range weather forecasts in the improvement of seasonal hydrologic prediction skill. Hydrol. Earth Syst. Sci., 16, 2825-2838, doi:10.5194/hess-16-2825-2012.

— J. Sheffield, E. F. Wood, and D. P. Lettenmaier, 2013: On the sources of global land surface hydrologic predictability. Hydrol. Earth Syst. Sci., 17, 2781-2796, doi:10.5194/hess-17-2781-2013.
Shuttleworth, W. J., 1999: Evaporation. Handbook of Hydrology, D. R. Maidment, Ed., McGraw-Hill, 4.1-4.53.

Sikka, D. R., 2009: Two decades of medium-range weather forecasting in India: National Centre for Medium-Range Weather Forecasting. COLA Tech. Rep. 276, 100 pp. [Available online at ftp://cola.gmu.edu/pub/ctr/CTR276_ms.pdf.]

Srivastava, A. K., M. Rajeevan, and S. R. Kshirsagar, 2009: Development of a high resolution daily gridded temperature data set (1969-2005) for the Indian region. Atmos. Sci. Lett., 10, 249-254, doi:10.1002/asl.232.

Tian, D., and C. J. Martinez, 2014: The GEFS-based daily reference evapotranspiration (ETo) forecast and its implication for water management in the southeastern United States. J. Hydrometeor., 15, 1152-1165, doi:10.1175/JHM-D-13-0119.1.

Wei, M., Z. Toth, R. Wobus, and Y. Zhu, 2008: Initial perturbations based on the ensemble transform (ET) technique in the NCEP global operational forecast system. Tellus, 60A, 62-79, doi:10.1111/j.1600-0870.2007.00273.x.

Wilks, D., 2006: Statistical Methods in the Atmospheric Science. 2nd ed. Academic Press, 684 pp.

Wood, A. W., E. P. Maurer, A. Kumar, and D. Lettenmaier, 2002: Long-range experimental hydrologic forecasting for the eastern United States. J. Geophys. Res., 107, 4429, doi:10.1029/ 2001JD000659.

World Meteorological Organization, 2012: Guidelines on ensemble prediction systems and forecasting. WMO-1091, $23 \mathrm{pp}$. [Available online at http://www.wmo.int/pages/prog/www/ Documents/1091_en.pdf.]

Wu, H., R. F. Adler, Y. Tian, G. J. Huffman, H. Li, and J. Wang, 2014: Real-time global flood estimation using satellitebased precipitation and coupled land surface and routing model. Water Resour. Res., 50, 2693-2717, doi:10.1002/ 2013WR014710. 